\title{
On Infinitely generated Fuchsian groups of the Loch Ness monster, the Cantor tree and the Blooming Cantor tree
}

https://doi.org/10.1515/coma-2020-0004

Received August 28, 2019; accepted October 31, 2019

\begin{abstract}
In this paper, for a non-compact Riemman surface $S$ homeomorphic to either: the Infinite Loch Ness monster, the Cantor tree and the Blooming Cantor tree, we give a precise description of an infinite set of generators of a Fuchsian group $\Gamma<P S L(2, \mathbb{R})$, such that the quotient space $\mathbb{H} / \Gamma$ is a hyperbolic Riemann surface homeomorphic to $S$. For each one of these constructions, we exhibit a hyperbolic polygon with an infinite number of sides and give a collection of Mobius transformations identifying the sides in pairs.
\end{abstract}

Keywords: Infinite Loch Ness Monster, Cantor tree, Blooming Cantor tree, Geometric Schottky groups, Noncompact surfaces

MSC: $20 \mathrm{H} 10,57 \mathrm{NO}, 57 \mathrm{~N} 16$

\section{Introduction}

A classical problem during the 19th century, in which several authors had been focused e.g., Klein, Schwarz, and Poincare among others, known as the uniformization problem, [1], said that: being $S$ a Riemann surface, find all domains $\widetilde{S} \subset \widehat{\mathbb{C}}$ and holomorphic functions $t: \widetilde{S} \rightarrow S$ such that at each point $p \in S, t$ is a local uniformizing variable at $p$. Equivalently, from the point of view of the Covering Spaces theory, there is a topological disc $B \subset S$ with center $p$ such that the restriction of $t$ to each component of $t^{-1}(B)$ is a homeomorphism. It will means that the triplet $(\widetilde{S}, S, t)$ is a covering space with base space $S$, total space $\widetilde{S}$, and holomorphic projection $t$. For $S$ being compact, it holds the first version of the Uniformization Theorem due to Klein.

Theorem 1.1. [6] Let $S$ be any compact Riemann surface of genus $\geq 2$. There exists a discrete subgroup $\Gamma$ of $\operatorname{PSL}(2, \mathbb{R})$ acting freely and properly on $\mathbb{H}$ such that $S$ is isomorphic to the quotient $\mathbb{H} / \Gamma$. In other words, the universal covering surface of $S$ is holomorphically isomorphic to $\mathbb{H}$.

However, the twenty-second of the Hilbert's problems proposes a major challenge, to find one uniformization being $\widetilde{S}$ simply connected. The answer to this problem is known as the Uniformization Theorem, which says:

Theorem 1.2. [2] Every simply connected Riemann Surface is conformally equivalent to either the Riemann sphere $\hat{\mathbb{C}}$ or the complex plane $\mathbb{C}$ or the unit disc $\Delta$. Let $S$ be a Riemann surface, let $\widetilde{S} \in\{\hat{\mathbb{C}}, \mathbb{C}, \Delta\}$ be its universal covering Riemann surface and $\Gamma<\operatorname{Aut}(\widetilde{S})$ be the cover group of $S$. Then

1. $S$ is conformally equivalent to $\widetilde{S} / \Gamma$;

\footnotetext{
^Corresponding Author: John A. Arredondo: Fundación Universitaria Konrad Lorenz, CP. 110231, Bogotá, Colombia, E-mail: alexander.arredondo@konradlorenz.edu.co

Camilo Ramurez Maluendas: Universidad Nacional de Colombia, Sede Manizales, Manizales, Colombia, E-mail: camramirezma@unal.edu.co
} 
2. $\Gamma$ is a Möbius group which acts discontinuously on $\widetilde{S}$;

3. Apart from the identity, the elements of $\Gamma$ have no fixed points in $\widetilde{S}$;

4. The cover group $\Gamma$ is isomorphic to $\pi(S)$.

From this valuable result we hold the following specific application: the complex plane $\mathbb{C}$ is the universal covering space of itself, the cylinder and the torus [7]. Thus when $\Gamma$, the cover group of $S$, has the identity as unique element, then the Riemann surface $\mathbb{C} / \Gamma$ is $\mathbb{C}$. If $\Gamma$ is generated by $z \mapsto z+1$, then the quotient space $\mathbb{C} / \Gamma$ is conformally equivalent to $\mathbb{C}-\{0\}$ (the cylinder). Finally, if $\Gamma$ is generated by $z \mapsto z+1$ and $z \mapsto z+\tau$, $\operatorname{Im}(\tau)>0$, then the quotient space $\mathbb{C} / \Gamma$ is a torus. On the other hand, the only Riemann surface $S$ which has as universal covering the sphere, is the sphere itself [7, Theorem 6.3.]. All surface except the previously listed have the unit disc $\Delta$ or equivalently the upper half plane $\mathbb{H}$ as their universal covering space. Moreover, if $S$ is a Riemann suface having universal covering space $\mathbb{H}$, then its cover group $\Gamma$ is a discrete subgroup of the isometries of $\mathbb{H}$. Hence, it is natural to ask. Given any non-compact Riemann surface S. Can one give a precise description of the set of generators of a Fuchsian group $\Gamma$ such that the quotient space $\mathbb{H} / \Gamma$ is a hyperbolic Riemann surface homeomorphic to $S$ ?

From the point of view of the Kerékjártó’s theorem of classification of non-compact surfaces (see e.g., [13], [19]), the topological type of any Riemann surface $S$ is given by its genus $g \in \mathbb{N} \cup\{\infty\}$ and a couple of nested, compact, metrizable and totally disconnected spaces $E n d s_{\infty} \subset \operatorname{Ends}(S)$, which are known as the ends space of $S$ and the ends of $S$, having (infinite) genus. Of all non-compact Riemann surfaces we focus on three of them: the Infinite Loch Ness monster (see Figure 1), the Cantor tree (see Figure 2-a) and the Blooming Cantor tree (see Figure 2-b). These surfaces have appeared in different reasearch in mathematics [4], [17].

Theorem 1.3. Let $\Gamma$ be the Fuchsian group generated by the of Möbius transformations $\left\{f_{n}, g_{n}: n \in \mathbb{Z}\right\}$, where

$$
f_{n}(z)=\frac{(8 n+4) z-(1+8 n(8 n+4))}{z-8 n}, g_{n}(z)=\frac{(8 n+6) z+(-1-(8 n+2)(8 n+6))}{z-(8 n+2)} \text {. }
$$

Then the hyperbolic Riemann surface $\mathbb{H} / \Gamma$ is homeomorphic to the Infinite Loch Ness monster.

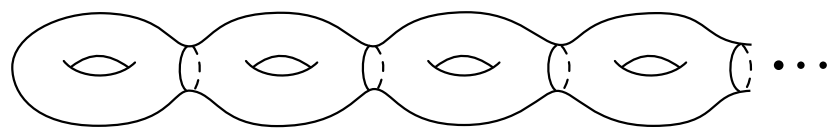

Figure 1: The Infinite Loch Ness monster.

Theorem 1.4. Let $\Gamma$ be the Fuchsian group generated by the union $\cup_{n \in \mathbb{N}} J_{n}$, where $J_{n}=$ $\left\{f_{n, k}: k \in\left\{0, \ldots, 2^{n-1}-1\right\}\right\}$ is formed by Möbius transformations

$$
f_{n, k}(z)=\frac{-2 \cdot\left(3^{n} \cdot 2+3+2 s_{2 k-1}\right) z+\frac{2^{2} \cdot\left(3^{n} \cdot 2+3+2 s_{2 k-1}\right)^{2}-1}{3^{n} \cdot 2^{2}}}{3^{n} \cdot 2^{2} z-2 \cdot\left(3^{n} \cdot 2+3+2 s_{2 k-1}\right)} .
$$

Then the hyperbolic Riemann surface $\mathbb{H} / \Gamma$ is homeomorphic to the Cantor tree. 


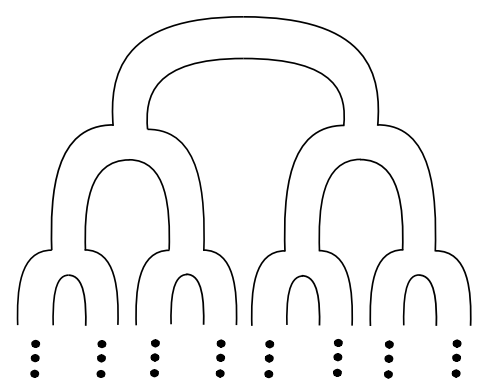

a. The Cantor tree.

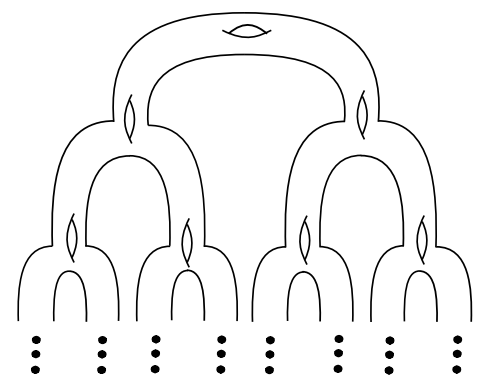

b. The Blooming Cantor tree.

Figure 2: Surfaces whose ends spaces are homeomorphic to the Cantor set.

Theorem 1.5. Let $\Gamma$ be the Fuchsian group generated by the union $\cup_{n \in \mathbb{N}} J_{n}$, where

$$
J_{n}=\left\{f_{n, k},\left(f_{n, k}\right)_{s, m}: k \in\left\{0, \ldots, 2^{n-1}-1\right\}, s \in\{1, \ldots, 4\}, m \in \mathbb{N}\right\},
$$

is composed by Möbius transformations ${ }^{1}$. Then the hyperbolic Riemann surface $\mathbb{H} / \Gamma$ is homeomorphic to the Blooming Cantor tree.

The paper is organized as follows: In section 2 we collect the principal tools used through the paper such as geometrical construction of the Cantor set, the classification theorem of non-compact and orientable surfaces, and a short introduction to the hyperbolic plane and Geometric Schottky groups. Finally, section 3 is dedicated to the proof of our main results.

\section{Preliminaries}

\subsection{Geometrical construction of the Cantor set}

For the Cantor set we begin its geometrical construction by removing the middle third started with the closed interval $I_{0}:=[1,2] \subset \mathbb{R}$. We denotes $I_{1}=\left[1,1+\frac{1}{3}\right] \cup\left[1+\frac{2}{3}, 2\right]$ the closed subset of $I_{0}$ held from $I_{0}$ by removing its middle third $\left(1+\frac{1}{3}, 1+\frac{2}{3}\right)$. The closed subset $I_{1} \subset I_{0}$ is the union of two disjoint closed intervals having length $\frac{1}{3}$. We denotes $I_{2}=\left[1,1+\frac{1}{9}\right] \cup\left[1+\frac{2}{9}, 1+\frac{1}{3}\right] \cup\left[1+\frac{2}{3}, 1+\frac{7}{9}\right] \cup\left[1+\frac{8}{9}, 2\right]$ be the closed subset of $I_{0}$ held from $I_{1}$ by removing its middle thirds $\left(1+\frac{1}{9}, 1+\frac{2}{9}\right)$ and $\left(1+\frac{7}{9}, 1+\frac{8}{9}\right)$ respectively. The closed subset $I_{2} \subset I_{0}$ is the union of four disjoint closed intervals having length $\frac{1}{3^{2}}$. We now construct inductively the closed subset $I_{n} \subset I_{0}$ from $I_{n-1}$ by removing its middle thirds.

On the other hand, we note that each positive integer number $k$ can be written in the binary form $k=$ $t_{0} \cdot 2^{0}+t_{1} \cdot 2^{1}+\ldots+t_{i} \cdot 2^{i}+\ldots+t_{m} \cdot 2^{m}$, where $t_{m}=1, t_{i} \in\{0,1\}$ for all $i \in\{0, \ldots, m\}$ and any $m \in \mathbb{N}$. Thus, we define $s_{k}$ as following

$$
s_{k}=2 \cdot t_{0} \cdot 3^{0}+2 \cdot t_{1} \cdot 3^{1}+\ldots+2 \cdot t_{i} \cdot 3^{i}+\ldots 2 \cdot t_{m} \cdot 3^{m} .
$$

Contrary, if $k=0$ then $s_{k}=0$.

Theorem 2.1. [16, Theorem 3.2.2]. For each $n \in \mathbb{N}, I_{n}=\bigcup_{k=0}^{2^{n}-1}\left[1+\frac{s_{k}}{3^{n}}, 1+\frac{s_{k}+1}{3^{n}}\right]$.

Remark 2.2. The closed subset $I_{n} \subset I_{0}$ is the union of $2^{n}$ disjoint closed intervals having length $\frac{1}{3^{n}}$. Moreover, $\left(1+\frac{s_{2 k}+1}{3^{n}}, 1+\frac{s_{2 k+1}}{3^{n}}\right)$ the middle thirds removed from $I_{n-1}$ also have length $\frac{1}{3^{n}}$, for each $k \in\left\{0, \ldots, 2^{n-1}-1\right\}$.

1 They will given explicitly in section 3.3. 
Therefore, the intersection of closed subset $2^{\omega}=\cap_{n \in \mathbb{N}} I_{n}$ is well-known as the Cantor set, which is the only totally disconnected, perfect compact metric space (up to homeomorphism, see [22, Corollary 30.4]).

\subsection{Ends spaces}

Definition 2.3. [9]. Let $X$ be a locally compact, locally connected, connected Hausdorff space, and let $U_{1} \supset$ $U_{2} \supset \cdots$ be an infinite nested sequence of non-empty connected open subsets of $X$, so that, the boundary of $U_{n}$ in $X$ is compact for every $n \in \mathbb{N}$,

$$
\bigcap_{n \in \mathbb{N}} \bar{U}=\emptyset,
$$

and for any compact subset $K$ of $X$ there is $l \in \mathbb{N}$ such that $U_{l} \cap K=\emptyset$. We shall denote the sequence $U_{1} \supset$ $U_{2} \supset \cdots$ as $\left(U_{n}\right)_{n \in \mathbb{N}}$. Two sequences $\left(U_{n}\right)_{n \in \mathbb{N}}$ and $\left(U_{n}^{\prime}\right)_{n \in \mathbb{N}}$ are equivalent if for any $l \in \mathbb{N}$ there is $k \in \mathbb{N}$ such that $U_{l} \supset U_{k}^{\prime}$ and, similarly, for any $n \in \mathbb{N}$ it exists $m \in \mathbb{N}$ such that $U_{n}^{\prime} \supset U_{m}$. The corresponding equivalence classes are called the topological ends of $X$. We will denote the space of ends by Ends $(X)$ and each equivalence class $\left[U_{n}\right]_{n \in \mathbb{N}} \in \operatorname{Ends}(X)$ is called an end of $X$.

For every non-empty open subset $U$ of $X$ in which its boundary $\partial U$ is compact, we define $U^{\star}=\left\{\left[U_{n}\right]_{n \in \mathbb{N}} \in\right.$ Ends $(X): U_{j} \subset U$ for some $\left.j \in \mathbb{N}\right\}$. The collection formed by all sets of the form $U \cup U^{\star}$, with $U$ open with compact boundary of $X$, forms a base for the topology of $X^{\prime}=X \cup E n d s(X)$.

Theorem 2.4. [18, Theorem 1.5]. Let $X^{\prime}=X \cup$ Ends(X) be the topological space defined above. Then,

1. The space $X^{\prime}$ is Hausdorff, connected and locally connected.

2. The space Ends $(X)$ is closed and has no interior points in $X^{\prime}$.

3. The space Ends $(X)$ is totally disconnected in $X^{\prime}$.

4. The space $X^{\prime}$ is compact.

5. If $V$ is any open connected set in $X^{\prime}$, then $V \backslash$ Ends $(X)$ is connected.

Ends of surfaces. An orientable surface $S$ is said to be planar if all of its compact subsurfaces are of genus zero. An end $\left[U_{n}\right]_{n \in \mathbb{N}}$ is called planar if there is $l \in \mathbb{N}$ such that $U_{l}$ is planar. The genus of a surface $S$ is the maximum of the genera of its compact subsurfaces. Remark that, if a surface $S$ has infinite genus, there is no finite set $\mathcal{C}$ of mutually non-intersecting simple closed curves with the property that $S \backslash \mathcal{C}$ is connected and planar. We define $E n d s_{\infty}(S) \subset E n d s(S)$ as the set of all ends of $S$ which are not planar (ends with infinity genus). It comes from the definition that $E n d s_{\infty}(S)$ forms a closed subspace of $E n d s(S)$.

Theorem 2.5 (Classification of non-compact and orientable surfaces, [13], [19]). Two non-compact and orientable surfaces $S$ and $S^{\prime}$ having the same genus are homeomorphic if and only if there is a homeomorphism $f: \operatorname{Ends}(S) \rightarrow \operatorname{Ends}\left(S^{\prime}\right)$ such that $f\left(\operatorname{Ends}_{\infty}(S)\right)=\operatorname{Ends}_{\infty}\left(S^{\prime}\right)$.

Proposition 2.6. [19, Proposition 3]. The space of ends of a connected surface $S$ is totally disconnected, compact, and Hausdorff. In particular, Ends(S) is homeomorphic to a closed subspace of the Cantor set.

Of all non-compact surfaces, our interest points at three of them. The first one is that surface which has infinite genus and only one end. It is called the Loch Ness monster (see Figure 1). Remark that a surface $S$ has only one end if and only if for all compact subset $K \subset S$ there is a compact $K^{\prime} \subset S$ such as $K \subset K^{\prime}$ and $S \backslash K^{\prime}$ is connected (see [21]). The other two remaining surfaces are the Cantor tree and the Blooming Cantor tree (see Figure 2), which have ends space homeomorphic to the Cantor set, although in the first one all ends are planar, while the ends of the second one are all not planar (see [10]).

Cantor binary tree. For every $n \in \mathbb{N}$ let $2^{n}=\prod_{i=1}^{n}\{0,1\}_{i}$ and let $\pi_{i}: 2^{n} \rightarrow\{0,1\}$ be the projection onto the $i$-th coordinate. We define $V=\left\{D_{s}: D_{s} \in 2^{n}\right.$ for some $\left.n \in \mathbb{N}\right\}$ and $E$ as the union of ((0), (1)) with the set 
$\left\{\left(D_{s}, D_{t}\right): D_{s} \in 2^{n}\right.$ and $D_{t} \in 2^{n+1}$ for some $n \in \mathbb{N}$, and $\pi_{i}\left(D_{s}\right)=\pi_{i}\left(D_{t}\right)$ for every $\left.i \in\{1, \ldots, n\}\right\}$. The infinite 3-regular tree with vertex set $V$ and edge set $E$ will be called the Cantor binary tree and denoted by $T 2^{\omega}$, (see Figure 3).

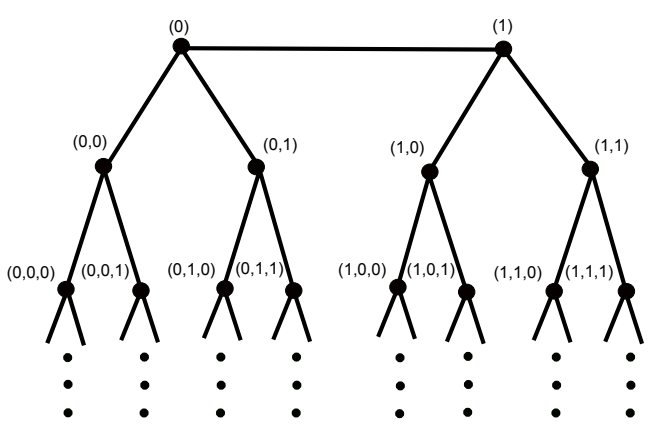

Figure 3: Cantor binary tree $T 2^{\omega}$.

Remark 2.7. Let $\left(v_{n}\right)_{n \in \mathbb{N}}$, where $v_{n} \in 2^{\omega}$ be an infinite simple path in $T 2^{\omega}$. If we define $V_{n}$ as the connected component of $T 2^{\omega} \backslash\left\{v_{n}\right\}$ such that $v_{n+1} \in V_{n}$, then $\left[V_{n}\right] \in \operatorname{Ends}\left(T 2^{\omega}\right)$ is completely determined by $\left(v_{n}\right)_{n \in \mathbb{N}}$. Hence, if we endow $\{0,1\}$ and $2^{\omega}=\prod_{i \in \mathbb{N}}\{0,1\}_{i}$ with the discrete and product topologies respectively, the map $f: \prod_{i \in \mathbb{N}}\{0,1\}_{i} \rightarrow \operatorname{Ends}\left(T 2^{\omega}\right)$, defines as $\left(x_{n}\right)_{n \in \mathbb{N}} \mapsto\left(v_{n}=\left(x_{1}, \ldots, x_{n}\right)\right)_{n \in \mathbb{N}}$, is a homeomorphism between the standard binary Cantor set and the space of ends of $T 2^{\omega}$. Note that each end $\left[V_{n}\right]_{n \in \mathbb{N}} \in \operatorname{Ends}\left(T 2^{\omega}\right)$ is determined by an infinite path $\left(v_{n}\right)_{n \in \mathbb{N}} \subset T 2^{\omega}$ such that $v_{n} \in 2^{n}$ for every $n \in \mathbb{N}$ and viceversa.

Remark 2.8. Sometimes we will abuse of notation to denote by $f\left(\left(x_{n}\right)_{n \in \mathbb{N}}\right)$ both: the end defined by the infinite path $\left(v_{n}=\left(x_{1}, \ldots, x_{n}\right)\right)_{n \in \mathbb{N}}$ and the infinite path itself in $T 2^{\omega}$.

\subsection{Hyperbolic plane}

The upper half-plane $\mathbb{H}=\{z \in \mathbb{C}: \operatorname{Im}(z)>0\}$ equipped with the riemannian metric $d s=\frac{\sqrt{d x^{2}+d y^{2}}}{y}$ is well known as either the hyperbolic or Lobachevski plane. The group $\operatorname{PSL}(2, \mathbb{R})$ is a subgroup of the isometries of $\mathbb{H}$ of index 2, where $\operatorname{PSL}(2, \mathbb{R})$ is composed by all fractional linear transformations or Möbius transformations, $z \mapsto \frac{a z+b}{c z+d}$, where the coefficients $a, b, c$ and $b$ are real numbers satisfying $a d-b c=1$.

Half-circles. Given the Möbius transformation $f \in P S L(2, \mathbb{R})$ with $c \neq 0$, then the half-circle $C(f)=\{z \in$ $\left.\mathbb{H}:|c z+d|^{-2}=1\right\}$ will be called the isometric circle of $f$ (see e.g., [15]). We note that $\frac{-d}{c} \in \mathbb{R}$ the center of $C(f)$ is mapped by $f$ onto the infinity point $\infty$. Further, $f$ sends the half-circle $C(f)$ onto $C\left(f^{-1}\right)=\{z \in \mathbb{H}$ : $\left.|-c z+a|^{-2}=1\right\}$ the isometric circle of the Möbius transformation $f^{-1}$.

Remark 2.9. The isometric circles $C(f)$ and $C^{-1}(f)$ have the same radius $r=|c|^{-1}$, and their respective centers are $\alpha=\frac{-d}{c}$ and $\alpha_{-1}=\frac{a}{c}$.

Let $C$ be a half-circle whose end points are in $\mathbb{R}$ and whose center is $\alpha \in \mathbb{R}$. There is a one-real-dimensional familiy of order two Möbius transformations in $\operatorname{PSL}(2, \mathbb{R})$ permuting these two end points. Each of them is uniquely determined by choosing a point on $C$ as its fixed point. In particular, there is exactly one of such a conformal involutions $f_{C}$ whose fixed point has real part $\alpha$, called the inversion in $C$. If $r>0$ is the radius of $C$, then $f_{C}$ is given as follows

$$
z \mapsto \frac{\frac{\alpha}{r} z+\left(\frac{-\alpha^{2}}{r}-r\right)}{\frac{z}{r}-\frac{\alpha}{r}} .
$$


The set $\check{C}(f)=\{z \in \mathbb{H}:|z-\alpha|<r\}$ is called the inside of $C$. Contrary, the set $\hat{C}(f):=\{z \in \mathbb{H}:|z-\alpha|>r\}$ is called the outside of $C$.

Remark 2.10. We let $L_{\alpha-2 r}$ and $L_{\alpha+2 r}$ be the two orthogonal straight lines to the real axis $\mathbb{R}$ through the points $\alpha-2 r$ and $\alpha+2 r$, respectively. Then the inversion $f_{C}$ sends $L_{\alpha-2 r}$ (analogously, $L_{\alpha+2 r}$ ) onto the half-circle whose ends points are $\alpha+\frac{r}{2}$ and $\alpha$ (respectively, $\alpha-\frac{r}{2}$ and $\alpha$ ), (see Figure 4). Given that $f_{C}$ is an element of PSL $(2, \mathbb{R})$, then for every $\epsilon<\frac{r}{2}$ the closed hyperbolic $\epsilon$-neighborhood of the half-circle $C$ does not intersect any of the hyperbolic geodesics $L_{\alpha-2 r}, f_{C}\left(L_{\alpha-2 r}\right), L_{\alpha+2 r}$, and $f_{C}\left(L_{\alpha+2 r}\right)$.

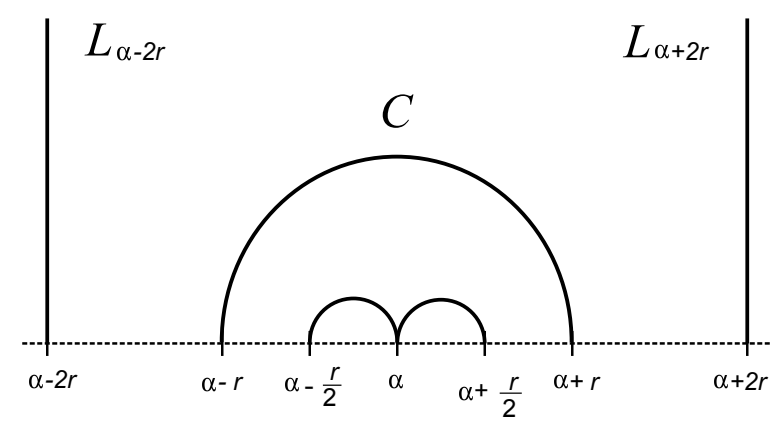

Figure 4: Inversion in $C$.

Lemma 2.11. Let $C_{1}$ and $C_{2}$ be two disjoint half-circles having centers and radius $\alpha_{1}, \alpha_{2} \in \mathbb{R}$, and $r_{1}, r_{2}>0$, respectively. Suppose that $\left|\alpha_{1}-\alpha_{2}\right|>\left(r_{1}+r_{2}\right)$, then for every $\epsilon<\frac{\min \left\{r_{1}, r_{2}\right\}}{2}$ the closed hyperbolic $\epsilon$-neighborhoods of the half-circles $C_{1}$ and $C_{2}$ are disjoint.

Proof. By hypothesis $\left|\alpha_{1}-\alpha_{2}\right|>\left(r_{1}+r_{2}\right)$, then the open strips $S_{1}=\left\{z \in \mathbb{H}: \alpha_{1}-2 r_{1}<\operatorname{Re}(z)<\alpha_{1}+2 r_{1}\right\}$ and $S_{2}=\left\{z \in \mathbb{H}: \alpha_{2}-2 r_{2}<\operatorname{Re}(z)<\alpha_{2}+2 r_{2}\right\}$ are disjoint. We remark that the half-circle $C_{i}$ belongs to the open strip $S_{i}$, for every $i \in\{1,2\}$. Further, $f_{C}$ the inversion in $C$,

$$
z \mapsto \frac{\frac{r_{2} z}{\sqrt{r_{1} r_{2}}}+\frac{\left(r_{1} \alpha_{2}-r_{2} \alpha_{1}\right)}{\sqrt{r_{1} r_{2}}}}{\frac{r_{1}}{\sqrt{r_{1} r_{2}}}}
$$

sends the open $\operatorname{strip} S_{1}$ onto the open strip $S_{2}$ and vice versa. We denote $r=\min \left\{r_{1}, r_{2}\right\}$. For each $\epsilon<\frac{r}{2}$, let $\bar{U}_{\epsilon}^{i}$ be the $\epsilon$-neighborhood of the half-circle $C_{i}$, with $i \in\{1,2\}$, by Remark $2.10 \bar{U}_{\epsilon}^{i} \subset S_{i}$. Given that $f_{C}$ is an element of $\operatorname{PSL}(2, \mathbb{R})$, then $f_{C}\left(\bar{U}_{\epsilon}^{1}\right)=\bar{U}_{\epsilon}^{2}$. Hence, for all $\epsilon<\frac{r}{2}, \bar{U}_{\epsilon}^{1} \cap \bar{U}_{\epsilon}^{2}=\emptyset$.

\subsection{Fuchsian groups and Fundamental region}

A subgroup $\Gamma$ of $P S L(2, \mathbb{R})$ is called Fuchsian if $\Gamma$ is discrete.

Definition 2.12. [15] Let $\Gamma<P S L(2, \mathbb{R})$ be a Fuchsian group. Let $R \subset \mathbb{H}$ be a let $D$ be its interior. We say that $R$ is a fundamental region for $\Gamma$ if the following properties hold:

(i) the union $\bigcup_{f \in \Gamma} f(R)=\mathbb{H}$;

(ii) the intersection of the interior sets $f(D) \cap D=\emptyset$, for each $f \in \Gamma \backslash\{I d\}$;

(iii) the boundary of $R$ (in the closure of $\mathbb{H}$, i.e., in $\mathbb{H} \cup \mathbb{R} \cup\{\infty\}$ consists of limits points of $\Gamma$, and a finite or countable collection of curves inside $\mathbb{H}$ (with the possible exception of its end points), called its sides; 
(iv) for each side $s$ of $R$ there is a side $s^{\prime}$ (not necessarilly different) and there is some $f_{s} \in \Gamma \backslash\{I d\}$ such that $f_{s}(s)=s^{\prime}$, called a side pairing, and $\left(s^{\prime}\right)^{\prime}=s$ and $f_{s^{\prime}}=f_{s}^{-1}$;

(v) if $s_{m}$ is a sequence of sides, then the spherical diameter of them goes to zero; the sides only accumulate at limit points of $\Gamma$;

(vi) only finitely many translates of D meet any compact subset of $\mathbb{H}$ (i.e., $R$ is locally finite).

If $\Gamma$ is a Fuchsian group such that none of its non-trivial elements fixes $\infty$ (i.e., $c \neq 0$ ), then the subset $R_{0}$ of $\mathbb{H}$ defined as follows

$$
R_{0}=\bigcap_{f \in \Gamma-\{I d\}} \overline{\hat{C}(f)} \subseteq \mathbb{H},
$$

is a fundamental domain for the group $\Gamma$ (see e.g., [8], [15, Theorem H.3], [11, Theorem 3.3.5]). The fundamental domain $R_{0}$ is well-known as the Ford region for $\Gamma$. On the other hand, we can get a Riemann surface from any Fuchsian group $\Gamma$. It is only necessary to define the action $\alpha: \Gamma \times \mathbb{H} \rightarrow \mathbb{H}$ as follows $(f, z) \mapsto f(z)$, which is proper and discontinuous (see [12, Theorem 8. 6]). Now, we define the subset

$$
K=\{w \in \mathbb{H}: f(w)=w \text { for some } f \in \Gamma-\{I d\}\} \subseteq \mathbb{H} .
$$

We note that the subset $K$ is countable and discrete. Moreover, the action $\alpha$ leaves invariant the subset $K$. Then the action $\alpha$ restricted to the hyperbolic plane $\mathbb{H}$ removing the subset $K$ is free, proper and discontinuous. Therefore, the quotient space $S=(\mathbb{H}-K) / \Gamma$ is well-defined and via $\pi:(\mathbb{H}-K) \rightarrow S$ the projection map $z \mapsto[z]$. It comes with a hyperbolic structure, it means, $S$ is a Riemann surface (see e.g., [14], [20, Theorem 18.2]).

Remark 2.13. If $R$ is a locally finite ${ }^{2}$ fundamental domain for the Fuchsian group $\Gamma$, then the quotient space $\mathbb{H} / \Gamma$ is homeomorphic to $R / \Gamma$ (see [3, Theorem 9.2.4 ]).

\subsection{Geometric Schottky groups}

Consider an open interval $A$ in the real line $\mathbb{R}$. Let $\partial A$ denote its boundary in $\mathbb{R}$, which consists of two points. The two points of $\partial A$ determine a geodesic $\gamma_{A}$ in $\mathbb{H}$. This geodesic in turn defines two closed half-planes in $\mathbb{H}$. The boundary at infinity of one of these two closed half-planes is equal to the closure $\bar{A}=A \cup \partial A$ and we will denote this closed half-plane by $\hat{A}$. In Figure 5 are depicted $A, \gamma_{A}$ and $\hat{A}$.

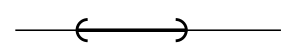

A
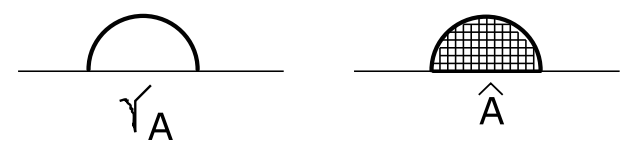

Figure 5: Objects $A, \gamma_{A}$ and $\hat{A}$.

A subset $I \subseteq \mathbb{Z}$ is called symmetric if it satisfies that $0 \notin I$ and for every $k \in I$ implies $-k \in I$.

Definition 2.14. [23, Definition 2] Let $\left\{A_{k}: k \in I\right\}$ be a family of open intervals with finite length in the real line $\mathbb{R}$, where $I$ is a symmetric subset of $\mathbb{Z}$ and let $\left\{f_{k}: k \in I\right\}$ be a subset of $\operatorname{PSL}(2, \mathbb{R})$. The pair $\mathfrak{U}\left(A_{k}, f_{k}, I\right)=$ $\left(\left\{A_{k}\right\},\left\{f_{k}\right\}\right)_{k \in I}$ is called a Schottky description ${ }^{3}$ if it satisfies the following conditions:

1. The closures subsets $\overline{A_{k}}$ in $\mathbb{C}$ are mutually disjoint.

2 In the sense due to Beardon [3, Definition 9.2.3].

3 The writer gives this definition to the Poincare disc and we use its equivalent to the half plane. 
2. For every $k \in I$, we denote as $C_{k}$ the half-circle whose ends points coincide to the ends points of $\overline{A_{k}}$, which is the isometric circle of $f_{k}$. Analogously, the half-circle $C_{-k}$ is the isometric circle of $f_{-k}=f_{k}^{-1}$.

3. There is an $\epsilon>0$ such that the closed hyperbolic $\epsilon$-neighborhood of the half-circles $C_{k}, k \in I$ are pairwise disjoint.

Note that condition (3) in Definition above implies that for each $k \in I$, the Möbius transformation $f_{k}$ is hyperbolic.

Definition 2.15. [23, Definition 3] A subgroup $\Gamma$ of $\operatorname{PSL}(2, \mathbb{R})$ is called Schottky type if there exists a Schottky description $\mathfrak{U}\left(A_{k}, f_{k}, I\right)$ such that the generated group by the set $\left\{f_{k}: k \in I\right\}$ is equal to $\Gamma$, i.e. we have

$$
\Gamma=\left\langle f_{k}: k \in I\right\rangle .
$$

Definition 2.16. [23, Definition 4] A subgroup $\Gamma$ of $\operatorname{PSL}(2, \mathbb{R})$ together a Schottky description $\mathfrak{U}\left(A_{k}, f_{k}, I\right)$ satisfying property 4 will be referred to as geometric Schottky group.

We note that any Schottky description $\mathfrak{U}\left(A_{k}, f_{k}, I\right)$ defines a Geometric Schottky group.

Proposition 2.17. [23, Proposition 4] Every Geometric Schottky group $\Gamma$ is a Fuchsian group.

The standard fundamental domain for the Geometric Schottky group $\Gamma$ having Schottky description $\mathfrak{U}\left(A_{k}, f_{k}, I\right)$ is the set $F(\Gamma)=\mathbb{H} \backslash\left(\bigcup_{k \in I} \hat{A}_{k}\right)$.

Proposition 2.18. [23, Proposition 2] The standard fundamental domain $F(\Gamma)$ is a fundamental domain for the Geometric Schottky group $\Gamma$.

\section{Main result}

The proof of our main results are based on the following sketch. First, we shall build explicitly a suitable family of mutually disjoint half circles $\mathcal{C}$. Then we shall define the set $J$ composed by the Möbius transformations having as isometric circles the elements of $\mathcal{C}$. Immediately, we will prove that $\Gamma$ the subgroup of $P S L(2, \mathbb{R})$ generated by $J$ is a Fuchsian group. Also, we show that the quotient space $\mathbb{H} / \Gamma$ is the desired non-compact surface.

\subsection{Proof Theorem 1.3}

Step 1. Building the group $\Gamma$. Given $\mathcal{C}$ the family composed by the half-circle with centers the even integers on the real line $\mathbb{R}$ and radius one (see Figure 6), we consider $\Gamma$ the Fuchsian subgroup of $\operatorname{PSL}(2, \mathbb{Z}$ ), which is generated by the set $\left\{f_{n}, g_{n}: n \in \mathbb{Z}\right\}$, where

$$
f_{n}(z)=\frac{(8 n+4) z-(1+8 n(8 n+4))}{z-8 n}, g_{n}(z)=\frac{(8 n+6) z+(-1-(8 n+2)(8 n+6))}{z-(8 n+2)} \text {. }
$$




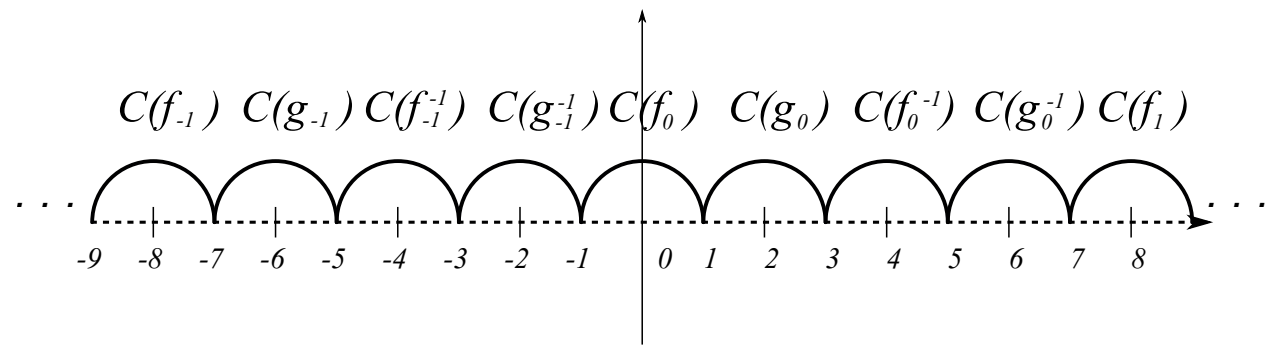

Figure 6: Family of half-circle $\mathcal{C}$.

For each $n \in \mathbb{Z}$, the Möbius transformations $f_{n}$ and $f_{n}^{-1}$ have as isometric circles the half-circles $C\left(f_{n}\right)$ and $C\left(f_{n}^{-1}\right)$ of $\mathcal{C}$, respectively, whose centers are $8 n$ and $8 n+4$, respectively. Analogously, the Möbius transformations $g_{n}(z)$ and $g_{n}^{-1}(z)$ have as isometric circles the half-circles $C\left(g_{n}\right)$ and $C\left(g_{n}^{-1}\right)$ of $\mathcal{C}$, respectively, whose centers are $8 n+2$ and $8 n+6$, respectively.

On the other hand, $\Gamma$ acts freely and properly discontinuously on $\mathbb{H}$, because the intersection of any two different elements belonged to $\mathcal{C}$ is either: empty or at infinity, it means, they meet at the same point in the real line $\mathbb{R}$. Then the quotient space $S=\mathbb{H} / \Gamma$ is a well-defined hyperbolic Riemann surface via the projection $\operatorname{map} \pi: \mathbb{H} \rightarrow S$.

Step 2. The desired surface. To end the proof we must prove that $S$ is the Infinite Loch Ness monster, i.e., it has infinite genus and only one end. The Ford region $R_{0}$ associated to $\Gamma$ (see Figure 7)

$$
\begin{aligned}
R_{0}= & \bigcap_{n \in \mathbb{Z}}\left(\overline{\hat{C}\left(f_{n}\right)} \cap \overline{\hat{C}\left(f_{n}^{-1}\right)} \cap \overline{\hat{C}\left(g_{n}\right)} \cap \overline{\hat{C}\left(g_{n}^{-1}\right)}\right), \\
= & \bigcap_{n \in \mathbb{Z}}\left(\left\{z \in \mathbb{H}:|z-8 n|^{-2} \leq 1\right\} \cap\left\{z \in \mathbb{H}:|-z+(8 n+4)|^{-2} \leq 1\right\}\right. \\
& \left.\cap\left\{z \in \mathbb{H}:|z-(8 n+2)|^{-2} \leq 1\right\} \cap\left\{z \in \mathbb{H}:|-z+(8 n+6)|^{-2} \leq 1\right\}\right),
\end{aligned}
$$

is a connected and locally finite subset of $\mathbb{H}$, having infinite hyperbolic area. Further, its boundary is $\mathcal{C}$, a family conformed by half-circles mutually disjoint. Given that $R_{0}$ the fundamental domain of $\Gamma$ is a noncompact Dirichlet region having infinite hyperbolic area, then the quotient space $S$ is also a non-compact hyperbolic surface with infinite hyperbolic area (see [12, Theorem 14.3]).

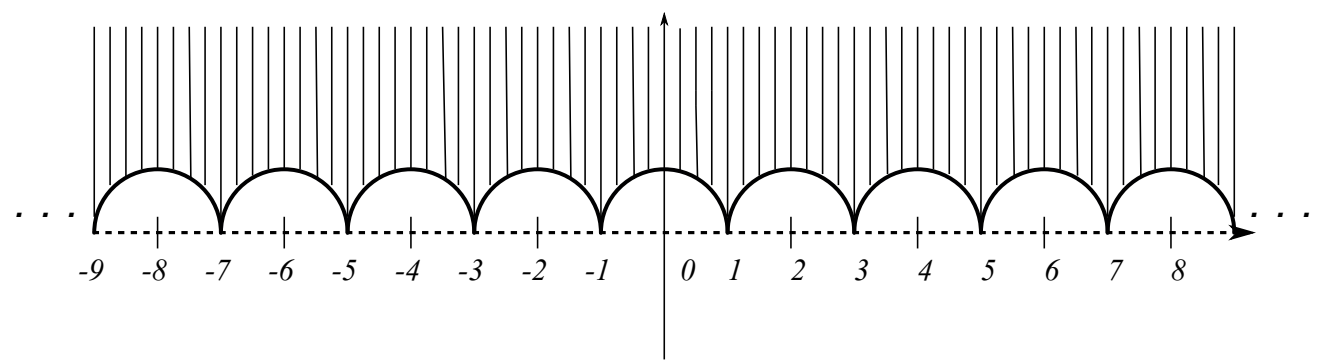

Figure 7: Ford region $R_{0}$ associated to $\Gamma$.

The surface $S$ has only one end. Let $K$ be a compact subset of $S$. We must prove that there is a compact subset $K \subset K^{\prime} \subset S$ such that $S \backslash K^{\prime}$ is connected. Given that the quotient $R_{0} / \Gamma$ is homeomorphic to $S$ we must suppose that there is a compact subset $B \subset R_{0}$ such that the projection map $\pi$ sends $B$ to $K$ i.e., $\pi(B)=K$. The hyperbolic plane $\mathbb{H}$ has exactly one end, then there exist two closed intervals $I_{1}, I_{2} \subset \mathbb{R}$ such that $B \subset$ $I_{1} \times I_{2} \subset \mathbb{H}$, and the difference $\mathbb{H} \backslash\left(I_{1} \times I_{2}\right)$ is connected. Then $\pi\left(\left(I_{1} \times I_{2}\right) \cap R_{0}\right)=K^{\prime}$ is a compact subset of $S$ such that $K \subseteq K^{\prime}$. We claim that $S \backslash K^{\prime}$ is connected. Let $[z]$ and [w] be two different points belonged to $S \backslash K^{\prime}$ we shall build a path in $S \backslash K^{\prime}$ joining both points. 
The following set are necessary to the proof. For every $x \in \mathbb{R} \backslash I_{1}$ we denote as $\gamma_{x}$ the perpendicular line to the real line, passing by $x$. For every $y>0$ with $y \notin I_{2}$ we consider the connected set $\gamma^{y}=\{z \in \mathbb{H}: \operatorname{Im}(z)=y\}$. Then the intersection $\gamma_{x} \cap\left(I_{1} \times I_{2}\right)=\emptyset$, and the projection map $\pi$ sends the set $\gamma_{x} \cap R_{0}$ into a connected subset of $S$. The intersection $\gamma^{y} \cap\left(I_{1} \times I_{2}\right)=\emptyset$, and the projection map $\pi$ sends the set $\gamma^{y} \cap R_{0}$ into a connected subset of $S$.

From the equivalent classes $[z] \neq[w] \in S \backslash K^{\prime}$ we can assume without loss of generality that $z, w \in$ $R_{0} \backslash\left(I_{1} \times I_{2}\right)$, then there exist two connected subsets $\gamma$ and $\gamma^{\prime}$ such that $z \in \gamma$ and $w \in \gamma^{\prime}$. If $\gamma \cap \gamma^{\prime} \neq \emptyset$ then the image of $\left(\gamma \cup \gamma^{\prime}\right) \cap R_{0}$ under $\pi$ is a connected subset belonged to $S \backslash K^{\prime}$ containing the points [z] and [w]. Oppositely, if $\gamma \cap \gamma^{\prime}=\emptyset$, then there is a connected subset $\gamma^{\star}$ such that $\gamma \cap \gamma^{\star} \neq \emptyset$ and $\gamma^{\prime} \cap \gamma^{\star} \neq \emptyset$. Consequently, the image of $\left(\gamma \cup \gamma^{\prime} \cup \gamma^{\star}\right) \cap R_{0}$ under $\pi$ is a connected subset belonged to $S \backslash K^{\prime}$ containing the points $[z]$ and $[w]$. This proves that the subset $S \backslash K^{\prime}$ is connected.

The surface $S$ has infinite genus. For every $n \in \mathbb{Z}$, we defined the subset

$$
S_{n}=\{z \in \mathbb{H}: 0<\operatorname{Im}(z)<3 \text { and }-1+8 n<\operatorname{Re}(z)<7+8 n\} \subset \mathbb{H} \text {. }
$$

The projection map $\pi$ sends the intersection $S_{n} \cap R_{0}$ into a subsurface with boundary $\widehat{S}_{n} \subset S$, which is homeomorphic to the torus punctured by only one point (see Figure 8). Furthermore, for any two different integers $m \neq n \in \mathbb{Z}$ the subsurfaces $\widehat{S}_{n}$ and $\widehat{S}_{m}$ are disjoint. Thus, we conclude that the hyperbolic surface $S$ has infinite genus.
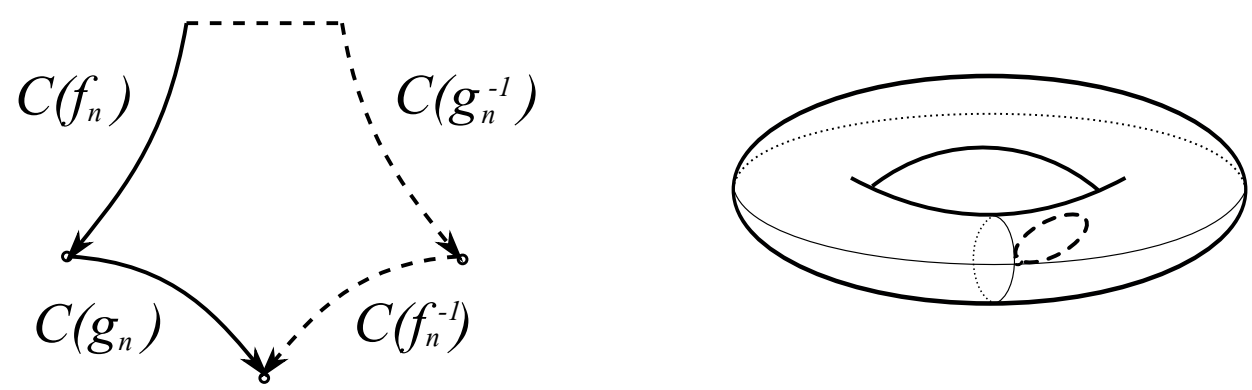

Figure 8: Subsurface $\widehat{S}_{n} \subset S$.

Corollary 3.1. The fundamental group of the Infinite Loch Ness monster is isomorphic to $\Gamma$.

\subsection{Proof Theorem 1.4}

Step 1. Build the group $\Gamma$. The following recursively construction of half-circles and Möbius transformations is necessary.

For $n=1$. Build the set $J_{1}$ containing exactly two Möbius transformations and the set $\bigodot_{1}$ composed by its respective isometric circles. We consider the closed intervals $I_{0}=[1,2]$ and $-I_{0}=[-2,-1]$ in the real line $\mathbb{R}$. We let $\hat{I}_{1}=\left[\frac{4+s_{0}}{3}, \frac{3+s_{1}}{3}\right]=\left[\frac{4}{3}, \frac{5}{3}\right],-\hat{I}_{1}=\left[-\frac{3+s_{1}}{3},-\frac{4+s_{0}}{3}\right]=\left[-\frac{5}{3},-\frac{4}{3}\right]$ be the middle thirds of $I_{0}$ and $-I_{0}$, respectively. If we remove $\hat{I}_{1}$ and $-\hat{I}_{1}$ of the closed intervals $I_{0}$ and $-I_{0}$ respectively, we hold the sets $I_{0} \backslash \hat{I}_{1}=I_{1}=\left[1, \frac{4}{3}\right] \cup\left[\frac{5}{3}, 2\right]$ and $\left(-I_{0}\right) \backslash\left(-\hat{I}_{1}\right)=-I_{1}=\left[-2,-\frac{5}{3}\right] \cup\left[-\frac{4}{3},-1\right]$. Given that the length of closed intervals $\hat{I}_{1}$ and $-\hat{I}_{1}$ is $\frac{1}{3}$, then we choose their respective middle points, which are given by

$$
\alpha_{1,1}=\frac{4+s_{0}}{3}+\frac{1}{3 \cdot 2}=\frac{3}{2}, \quad \alpha_{1,1}^{-1}=-\frac{4+s_{0}}{3}-\frac{1}{3 \cdot 2}=-\frac{3}{2} .
$$

Let $C\left(f_{1,1}\right)$ and $C\left(f_{1,1}^{-1}\right)$ be the two half-circles having centers $\alpha_{1,1}$ and $\alpha_{1,1}^{-1}$ respectively, and the same radius $r(1)=\frac{1}{3 \cdot 2^{2}}$, (see Figure 9). Now, we take the Möbius transformations

$$
f_{1,1}(z)=\frac{a_{1,1} z+b_{1,1}}{c_{1,1} z+d_{1,1}}, \quad f_{1,1}^{-1}(z)=\frac{d_{1,1} z-b_{1,1}}{-c_{1,1} z+a_{1,1}},
$$




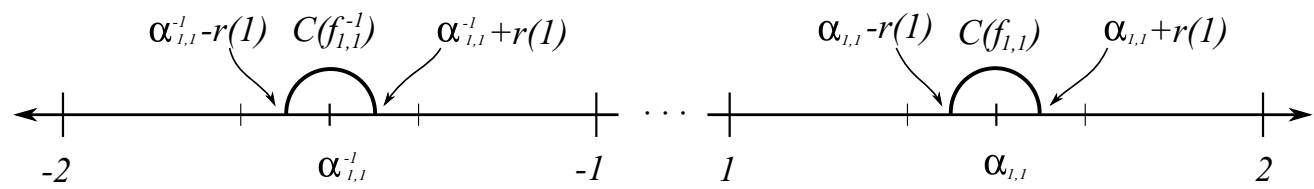

Figure 9: Half-circles $C\left(f_{1,1}\right)$ and $C\left(f_{1,1}^{-1}\right)$.

which have as isometric circles $C\left(f_{1,1}\right)$ and $C\left(f_{1,1}^{-1}\right)$, respectively. Using Remark 2.9 we have $c_{1,1}=\frac{1}{r(1)}=3 \cdot 2^{2}$, $d_{1,1}=-c_{1,1} \cdot \alpha_{1,1}=-18$, and $a_{1,1}=c_{1,1} \cdot \alpha_{1,1}^{-1}=-18$. Now, we substitute these values in the equation $a_{1,1} \cdot d_{1,1}-c_{1,1} \cdot b_{1,1}=1$ and computing we hold $b_{1,1}=\frac{323}{12}$. Finally, we define the sets

$$
J_{1}=\left\{f_{1,1}, f_{1,1}^{-1}\right\}, \quad \mathcal{C}_{1}=\left\{C\left(f_{1,1}\right), C\left(f_{1,1}^{-1}\right)\right\} .
$$

By construction, each Möbius transformations of $J_{1}$ is hyperbolic and the half-circles of $\mathcal{C}_{1}$ are pairwise disjoint.

For $n$. Build a set $J_{n}$ containing $2^{n}$ Möbius transformations and the set $\mathcal{C}_{n}$ composed by its respective isometric circles. We consider the closed subsets $I_{n-1}=\bigcup_{k=0}^{2^{n-1}-1}\left[\frac{3^{n-1}+s_{k}}{3^{n-1}}, \frac{3^{n-1}-s_{k}+1}{3^{n-1}}\right] \subseteq I_{0}$ and $-I_{n-1}=\bigcup_{k=0}^{2^{n-1}-1}\left[-\frac{3^{n-1}-s_{k}+1}{3^{n-1}},-\frac{3^{n-1}+s_{k}}{3^{n-1}}\right] \subseteq-I_{0}$ (see Theorem 2.1). For each $k \in\left\{0, \ldots, 2^{n-1}-1\right\}$ we let $\hat{I}_{n, k}=\left[\frac{3^{n}+S_{2 k-2}+1}{3^{n}}, \frac{3^{n}+s_{2 k-1}}{3^{n}}\right],-\hat{I}_{n, k}=\left[-\frac{3^{n}+S_{2 k-1}}{3^{n}},-\frac{3^{n}+s_{2 k-2}+1}{3^{n}}\right]$ be the middle thirds of the closed intervals $\left[\frac{3^{n-1}+s_{k-1}}{3^{n-1}}, \frac{3^{n-1}+s_{k-1}+1}{3^{n-1}}\right]$ and $\left[-\frac{3^{n-1}+s_{k-1}+1}{3^{n-1}},-\frac{3^{n-1}+s_{k-1}}{3^{n-1}}\right]$, respectively. If we remove $\bigcup_{k=0}^{2^{n-1}-1} \hat{I}_{n, k}$ and $\bigcup_{k=0}^{2^{n-1}-1}-\hat{I}_{n, k}$ of the closed subsets $I_{n-1}$ and $-I_{n-1}$ accordingly, we hold the sets

$$
\begin{gathered}
I_{n-1} \backslash\left(\bigcup_{k=0}^{2^{n-1}-1} \hat{I}_{n, k}\right)=I_{n}=\bigcup_{k=0}^{2^{n}-1}\left[\frac{3^{n}+s_{k}}{3^{n}}, \frac{3^{n}+s_{k}+1}{3^{n}}\right], \\
\left(-I_{n-1}\right) \backslash\left(\bigcup_{k=0}^{2^{n-1}-1}-\hat{I}_{n, k}\right)=-I_{n}=\bigcup_{k=0}^{2^{n}-1}\left[-\frac{3^{n}+s_{k}+1}{3^{n}},-\frac{3^{n}+s_{k}}{3^{n}}\right] .
\end{gathered}
$$

Given that for every $k \in\left\{0, \ldots, 2^{n-1}-1\right\}$ the length of the closed intervals $\hat{I}_{n, k}$ and $-\hat{I}_{n, k}$ is $\frac{1}{3^{n}}$, then we choose their respective middle points, which are given by

$$
\begin{aligned}
& \alpha_{n, k}=1+\frac{1+s_{2 k-1}}{3^{n}}+\frac{1}{3^{n} \cdot 2}=\frac{3^{n} \cdot 2+3+2 s_{2 k-1}}{3^{n} \cdot 2}, \\
& \alpha_{n, k}^{-1}=-1-\frac{1+s_{2 k-1}}{3^{n}}-\frac{1}{3^{n} \cdot 2}=-\frac{3^{n} \cdot 2+3+2 s_{2 k-1}}{3^{n} \cdot 2} .
\end{aligned}
$$

Hence, we let $C\left(f_{n, k}\right), C\left(f_{n, k}^{-1}\right)$ be the two half-circles having centers $\alpha_{n, k}$ and $\alpha_{n, k}^{-1}$ correspondingly, and the same radius $r(n)=\frac{1}{3^{n} \cdot 2^{2}}$ (see Figure 10). Now, for each $k \in\left\{0, \ldots, 2^{n-1}-1\right\}$ we take the Möbius transformations

$$
f_{n, k}(z)=\frac{a_{n, k} z+b_{n, k}}{c_{n, k} z+d_{n, k}}, \quad f_{n, k}^{-1}(z)=\frac{d_{n, k} z-b_{n, k}}{-c_{n, k} z+a_{n, k}},
$$

which have as isometric circles $C\left(f_{n, k}\right)$ and $C\left(f_{n, k}^{-1}\right)$, respectively. Using Remark 2.9 we have $c_{n, k}=\frac{1}{r(n)}=3^{n} \cdot 2^{2}$, $d_{n, k}=-c_{n, k} \cdot \alpha_{n, k}=-2 \cdot\left(3^{n} \cdot 2+3+2 s_{2 k-1}\right), a_{n, k}=c_{n, k} \cdot \alpha_{n, k}^{-1}=-2 \cdot\left(3^{n} \cdot 2+3+2 s_{2 k-1}\right)$. Now, we substitute these values in the equation $a_{n, k} \cdot d_{n, k}-c_{n, k} \cdot b_{n, k}=1$ and computing we hold $b_{n, k}=\frac{2^{2} \cdot\left(3^{n} \cdot 2+3+2 s_{2 k-1}\right)^{2}-1}{3^{n} \cdot 2^{2}}$.

Finally, we define the sets

$$
\begin{aligned}
& J_{n}=\left\{f_{n, k}, f_{n, k}^{-1}: k \in\left\{0, \ldots, 2^{n-1}-1\right\}\right\}, \\
& \mathcal{C}_{n}=\left\{C\left(f_{n, k}\right), C\left(f_{n, k}^{-1}\right): k \in\left\{0, \ldots, 2^{n-1}-1\right\}\right\} .
\end{aligned}
$$

By construction, each Möbius transformation of $J_{n}$ is hyperbolic and the half-circles of $\mathcal{C}_{n}$ are pairwise disjoint. 


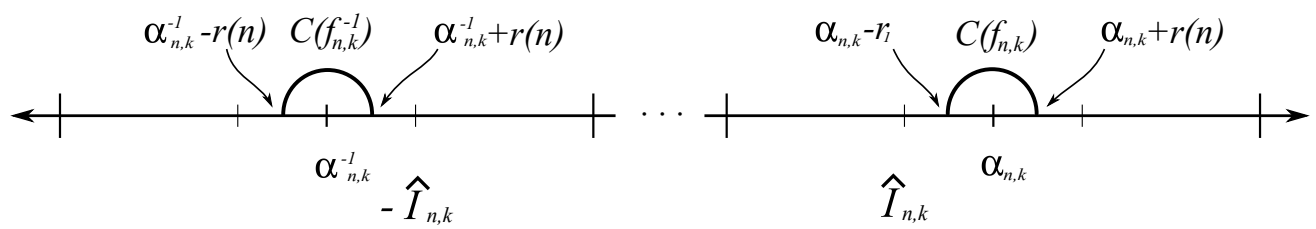

Figure 10: Half circles $C\left(f_{n, k}\right)$ and $C\left(f_{n, k}^{-1}\right)$.

From the previous recursive construction of Möbius transformations and half-circles, we define the sets

$$
J=\bigcup_{n \in \mathbb{N}} J_{n}, \quad \mathcal{C}=\bigcup_{n \in N} \mathcal{C}_{n} .
$$

Hence, we denote the subgroup of $\operatorname{PSL}(2, \mathbb{R})$ generated by the set $J$ by $\Gamma$. We observe that by construction each Möbius transformation of $J$ is hyperbolic and the half-circles of $\mathcal{C}$ are pairwise disjoint.

Step 2. The group $\Gamma$ is a Fuchsian group. In order to $\Gamma$ will be a Geometric Schottky group, we shall define a Schottky description for it. Hence, by Proposition 2.17 we will conclude that $\Gamma$ is Fuchsian.

The elements belonged to the set $J$ can be indexed by a symmetric subset of $\mathbb{Z}$. Merely, we consider $P=$ $\left\{p_{n}\right\}_{n \in \mathbb{N}}$ the subset of all the prime numbers. Then it is easy to check that the map $\psi: J \rightarrow \mathbb{Z}$ defined by

$$
f_{n, k} \mapsto p_{n}^{k}, \quad f_{n, k}^{-1} \mapsto-p_{n}^{k}, \quad \text { (for every } n \in \mathbb{N} \text { and } k \in\left\{0, \ldots, 2^{n-1}-1\right\} \text { ), }
$$

is well-defined and injective. The image of $J$ under $\psi$ is a symmetric subset of $\mathbb{Z}$, which we denote as $I$. Given that for each element $k$ belonged to $I$ there is a unique transformation $f \in J$ such that $\psi(f)=k$, then we label the map $f$ as $f_{k}$ and its respective isometric circle as $C\left(f_{k}\right)$. Hence, we re-write the sets $J$ and $\mathcal{C}$ as follows

$$
J=\left\{f_{i}\right\}_{i \in I}, \quad \mathcal{C}=\left\{C\left(f_{i}\right)\right\}_{i \in I} .
$$

On the other hand, we define the set $\left\{A_{i}\right\}_{i \in I}$ where $A_{i}$ is the straight segment in the real line $\mathbb{R}$ whose ends points coincide to the ends points at the infinite of the half-circle $C\left(f_{i}\right)$ (see equation (10)). We claim that the pair $\left(\left\{A_{i}\right\},\left\{f_{i}\right\}\right)_{i \in I}$ is a Schottky description. By the inductive construction of the set $J:=\left\{f_{i}\right\}_{i \in I}$ described above, it is immediate that the pair $\left(\left\{A_{i}\right\},\left\{f_{i}\right\}\right)_{i \in I}$ satisfies the conditions 1 and 2 of the Definition 2.14. Thus, we must only prove that the condition 3 of Definition 2.14 is done. For any $i \in I$, we denote as $\alpha_{i}$ and $r_{i}$ the center and radius of $C\left(f_{i}\right)$. Hence, we define the open strip $S_{i}=\left\{z \in \mathbb{H}: \alpha_{i}-2 r_{i}<\operatorname{Re}(z)<\alpha_{i}+2 r_{i}\right\}$ associated to $f_{i}$ (see Figure 4). We note that $C\left(f_{i}\right) \subset S_{i}$ and for any two different transformations $f_{i} \neq f_{j} \in J$ its respective open strips associated $S_{i}$ and $S_{j}$ are disjoint. Further, by construction of the family $\mathcal{C}$ (see equation (10)) there exist a transformation $f_{k} \in J$ such that $r_{k}$, the radius of its isometric circle $C\left(f_{k}\right)$ satisfies $r_{i} \leqslant r_{k}$ for all $i \in I$. Moreover, by Remark 2.10 it follows that $B_{\epsilon}^{k}$, the closed hyperbolic $\epsilon$-neighborhood of the half-circle $C\left(f_{k}\right)$ is belonged to the open strip $S_{k}$, choosing $\epsilon<\frac{r_{k}}{2}$. Since, for all $i \in I$ we have $\left|\alpha_{k}-\alpha_{i}\right|>\left(r_{k}+r_{i}\right)$, by Lemma 2.11 for every $\epsilon<\frac{\max \left\{r_{k}, r_{i}\right\}}{2}=\frac{r_{k}}{2}$ the closed hyperbolic $\epsilon$-neighborhoods $B_{\epsilon}^{k}$ and $B_{\epsilon}^{i}$ of the half circle $C\left(f_{k}\right)$ and $C\left(f_{i}\right)$ are disjoint. Even more, this lemma assures that $B_{\epsilon}^{i} \subseteq S_{i}$, for all $i \in I$. This implies that condition 3 is done. Therefore, we conclude that the pair $\left(\left\{A_{i}\right\},\left\{f_{i}\right\}\right)_{i \in I}$ is a Schottky description.

Step 3. Holding the surface called the Cantor tree. The Geometric Schottky group $\Gamma$ acts freely and properly discontinuously on the hyperbolic plane $\mathbb{H}$, because the intersection of any two different elements of $\mathcal{C}$ is empty. Then the quotient space $S:=\mathbb{H} / \Gamma$ is well-defined and through the projection map $\pi: \mathbb{H} \rightarrow S$ defines as $z \mapsto[z]$ is a hyperbolic surface. To end the proof we shall show that $S$ is the Cantor tree i.e., its ends space is homeomorphic to the Cantor set having all ends planar. To prove this, we will describe the ends space of $S$ using the property of $\sigma$-compact of $S$ and showing that there is a homeomorphism $f$ from the ends spaces of the Cantor binary tree $\operatorname{Ends}\left(T 2^{\omega}\right)$ onto the ends space $\operatorname{Ends}(S)$.

Remark 3.2. We let $F(\Gamma)=\bigcap_{i \in I} \overline{\hat{C}\left(f_{i}\right)} \subset \mathbb{H}$ be the standard fundamental domain of $\Gamma$. Regarding Proposition 2.18 it is a fundamental domain for $\Gamma$ having the following properties. 
1. It is connected and locally finite having infinite hyperbolic area. Further, its boundary is composed by the family of half-circle $\mathrm{C}$ (see equation (10)). In other words, it consists of infinitely many hyperbolic geodesic with ends points at infinite and mutually disjoint.

2. It is a non-compact Ford region and the quotient $F(\Gamma) / \Gamma$ is homeomorphic to $S$, then the quotient space $S$ is also a non-compact hyperbolic surface with infinite hyperbolic area (see [12, Theorem 14.3]). We note that by construction, the surface $S$ does not have genus i.e., its ends are planar.

Since surfaces are $\sigma$-compact space, for $S$ there is an exhaustion of $S=\bigcup_{n \in \mathbb{N}} K_{n}$ by compact sets whose complements define the ends spaces of the surface $S$. More precisely;

For $n=1$. We consider the radius $r(1)=\frac{1}{3 \cdot 2^{2}}$ given in the recursive construction of $\Gamma$ and define the compact subset $\tilde{K}_{1}=\{z \in \mathbb{H}:-2 \leq \operatorname{Re}(z) \leq 2$, and $r(1) \leq \operatorname{Im}(z) \leq 1\}$. The image $\pi\left(\tilde{K}_{1} \cap F(\Gamma)\right)=K_{1}$ is a compact subset of $S$ and, the difference $S \backslash K_{1}$ consists of two connected components whose closure in $S$ is non-compact, but they have compact boundary. Hence, we can write $S \backslash K_{1}=U_{0} \sqcup U_{1}$. The set of connected components of $S \backslash K_{1}$ and the set $2^{1}=\{0,1\}$ are equipotent.

For $n$. We consider the radius $r(n)=\frac{1}{3^{n} \cdot 2^{2}}$ given in the recursive construction of $\Gamma$ and define the compact subset $\tilde{K}_{n}=\{z \in \mathbb{H}:-(n+1) \leq \operatorname{Re}(z) \leq n+1$, and $r(n) \leq \operatorname{Im}(z) \leq n\}$. By construction $\tilde{K}_{n-1} \subset \tilde{K}_{n}$ and the image $\pi\left(\tilde{K}_{n} \cap F(\Gamma)\right)=K_{n}$ is a compact subset of $S$ just as $K_{n-1} \subset K_{n}$. By definition of $\tilde{K}_{n}$ the difference $S \backslash K_{n}$ consists of $2^{n}$ connected components whose closure in $S$ is non-compact, but they have compact boundary. Moreover, for every $l$ in the set $2^{n-1}$ there exist exactly two connected components of $S \backslash K_{n-1}$ contained in $U_{l} \subset S \backslash K_{n-1}$. Hence, we can write $S \backslash K_{n}=\bigsqcup_{l \in 2^{n-1}}\left(U_{l, 0} \sqcup U_{l, 1}\right)$, being $U_{l, 0}, U_{l, 1} \subset U_{l}$ for every $l \in 2^{n-1}$. The set of connected components of $S \backslash K_{n}$ and the set $2^{n}=\prod_{i=1}^{n}\{0,1\}_{i}$ are equipotent.

This recursive construction induces the desired numerable family of increasing compact subsets $K_{1} \subset$ $K_{2} \subset \ldots$ covering the surface $S$, i.e., $S=\bigcup_{n \in \mathbb{N}} K_{n}$. Thus, the ends space of $S$ is composed by all sequences $\left(U_{l_{n}}\right)_{n \in \mathbb{N}}$ such that $U_{l_{n}} \subset S \backslash K_{n}$ and $U_{l_{n}} \supset U_{l_{n+1}}$, for each $n \in \mathbb{N}$. Further, $l_{n} \in 2^{n}, l_{n+1} \in 2^{n+1}$ such that $\pi_{i}\left(l_{n}\right)=\pi_{i}\left(l_{n+1}\right)$ for every $i \in\{1, \ldots, n\}$, (see subsection Cantor binary tree). It is easy to check that the $\operatorname{map} f: \operatorname{Ends}\left(T 2^{\omega}\right) \rightarrow \operatorname{Ends}(S)$ defines as $\left(v_{i}\right)_{i \in \mathbb{N}} \mapsto\left[U_{v_{i}}\right]_{i \in \mathbb{N}}$ is well-defined. We now prove that is a closed continuous bijection, hence a homeomorphism.

Injectivity. Consider two different infinite sequences $\left(v_{n}\right)_{n \in \mathbb{N}} \neq\left(v_{n \in \mathbb{N}}^{\prime}\right)$ of the graph $T 2^{\omega}$. Then there is $N \in \mathbb{N}$ such that for all $m>N$ we have $v_{m} \neq v_{m}^{\prime}$. This implies that $U_{v_{m}} \cap U_{v_{m}^{\prime}}=\emptyset$, hence $f\left(\left(v_{n}\right)_{n \in \mathbb{N}}\right)=\left[U_{v_{n}}\right]_{n \in \mathbb{N}} \neq$ $\left[U_{v_{n}^{\prime}}\right]_{n \in \mathbb{N}}=f\left(\left(v_{n}^{\prime}\right)_{n \in \mathbb{N}}\right)$.

Surjectivity. Consider an end $\left[W_{n}\right]_{n \in \mathbb{N}}$ of $E n d s(S)$, then for each $l \in \mathbb{N}$ there is a $W_{n_{l}}$ of the sequence $\left(W_{n}\right)_{n \in \mathbb{N}}$ such that $K_{l} \cap W_{n_{l}}=\emptyset$. Hence, $W_{n_{l}}$ is contained to $U_{s_{l}}$ a connected component of $S \backslash K_{l}$ such that $s_{l} \in 2^{l}$. Given that $W_{n_{l}}$ is a connected open such that $W_{n_{l-1}} \supseteq W_{n_{l}}$ then $U_{s_{l-1}} \supset U_{s_{l}}$ for every $l \in \mathbb{N}$ i.e., the sequences $\left(W_{n}\right)_{n \in \mathbb{N}}$ and $\left(U_{s_{l}}\right)_{l \in \mathbb{N}}$ define the same end in $S$ i.e., $\left[W_{n}\right]_{n \in \mathbb{N}}=\left[U_{s_{l}}\right]_{l \in \mathbb{N}}$. This implies that the sequence $\left(s_{l}\right)_{l \in \mathbb{N}}$ is an infinite path in $T 2^{\omega}$ defining an end of $T 2^{\omega}$ and $f$ sends the sequence $\left(s_{l}\right)_{l \in \mathbb{N}}$ into the ends $\left[W_{n}\right]_{n \in \mathbb{N}}=\left[U_{s_{l}}\right]_{l \in \mathbb{N}}$.

The map $f$ is continuous. Given an end $\left(v_{n}\right)_{n \in \mathbb{N}} \in \operatorname{Ends}\left(T 2^{\omega}\right)$ and an open subset $W \subset S$ whose boundary is compact such that the basic open $W^{\star}$ contains the point $f\left(\left(v_{n}\right)_{n \in \mathbb{N}}\right)=\left[U_{v_{n}}\right]_{n \in \mathbb{N}}$, we must prove that there is an open subset $Z \subset T 2^{\omega}$ such that the basic open $Z^{\star}$ contains the point $\left(v_{n}\right)_{n \in \mathbb{N}}$ and $f\left(Z^{\star}\right) \subset W^{\star}$. Hence $\left[U_{v_{n}}\right]_{n \in \mathbb{N}} \in W^{\star}$ and there is $m \in \mathbb{N}$ such that $U_{v_{m}} \subset W$. If we remove the vertex $v_{m}$ of the graph $T 2^{\omega}$ the subset $T 2^{\omega} \backslash\left\{v_{m}\right\}$ consists of exactly three open connected components whose closure in $T 2^{\omega}$ are noncompact, but has compact boundary. We denote as $Z$ the connected component of $T 2^{\omega}$ having the infinite sequence $\left(v_{m+i}\right)_{i \in \mathbb{N}}$. Then the subset $Z^{*} \subset \operatorname{Ends}\left(T 2^{\omega}\right)$ is the required basic open, which contains the points $\left(v_{n}\right)_{n \in \mathbb{N}}$ and $f\left(Z^{*}\right) \subset W^{*}$.

The map $f$ is closed. It holds because $f$ is a continuous bijection from a compact space into a Hausdorff space (see [5, Theorem 2.1]). Hence $f$ is a homeomorphism.

Corollary 3.3. The fundamental group of the Cantor tree is isomorphic to $\Gamma$. 


\subsection{Proof Theorem 1.5}

\section{Step 1. Building the group $\Gamma$.}

For $n=1$. Building the set $J_{1}$ containing infinitely countable Möbius transformation and the set $\mathcal{C}_{1}$ composed by its respective isometric circles. We let $C\left(f_{1,1}\right)$ and $C\left(f_{1,1}^{-1}\right)$ be two half-circles having centers $\alpha_{1,1}$ and $\alpha_{1,1}^{-1}$, respectively (as in equation (6)), and the same radius $r(1)=\frac{1}{6 \cdot 3 \cdot 2}$ (see Figure 11). Now, we consider the Möbius transformations

$$
f_{1,1}(z)=\frac{a_{1,1} z+b_{1,1}}{c_{1,1} z+d_{1,1}}, \quad f_{1,1}^{-1}(z)=\frac{d_{1,1} z-b_{1,1}}{-c_{1,1} z+a_{1,1}},
$$

which has as isometric circles $C\left(f_{1,1}\right)$ and $C\left(f_{1,1}^{-1}\right)$, respectively. By Remark 2.9 we have $a_{1,1}=-54, c_{1,1}=36$, $d_{1,1}=-54$. From the relation $a_{1,1} \cdot d_{1,1}-b_{1,1} \cdot c_{1,1}=1$ we hold $b_{1,1}=\frac{2915}{36}$.

By construction the Möbius transformations $f_{1,1}$ and $f_{1,1}^{-1}$ are hyperbolic and the half-circles $C\left(f_{1,1}\right)$ and $C\left(f_{1,1}^{-1}\right)$ are pairwise disjoint.

Now, we shall build sequences of half-circles at the left and at the right of $C\left(f_{1,1}\right), C\left(f_{1,1}^{-1}\right)$, whose radius converge to zero. Each sequence will have associated a suitable sequence of Möbius transformations as follows.

Part I. Building sequences of half-circles at the left of $C\left(f_{1,1}\right)$ and at right of $C\left(f_{1,1}^{-1}\right)$. We divide the closed intervals $\hat{I}_{1,1}$ and $-\hat{I}_{1,1}{ }^{4}$ into six as is shown in Figure 11, and we consider the second sixth closed subintervals of them, which are given by $L_{1,1}=\left[\frac{4+s_{0}}{3}+\frac{1}{3 \cdot 6}, \frac{4+s_{0}}{3}+\frac{2}{3 \cdot 6}\right]=\left[\frac{25}{18}, \frac{26}{18}\right]$ and $-L_{1,1}=\left[-\frac{26}{18},-\frac{25}{18}\right]$, respectively. Now, we write $L_{1,1}$ and $-L_{1,1}$ as the union of closed subintervals following

$$
\begin{aligned}
L_{1,1} & =\bigcup_{m \in \mathbb{N}}\left(L_{1,1}\right)_{m}=\bigcup_{m \in \mathbb{N}}\left[\frac{25+6 s_{0}}{3 \cdot 6}+\frac{1}{3 \cdot 6 \cdot 2^{m}}, \frac{25+6 s_{0}}{3 \cdot 6}+\frac{1}{3 \cdot 6 \cdot 2^{m-1}}\right] \\
& =\bigcup_{m \in \mathbb{N}}\left[\frac{25 \cdot 2^{m}+1}{18 \cdot 2^{m}}, \frac{25 \cdot 2^{m-1}+1}{18 \cdot 2^{m-1}}\right], \\
-L_{1,1} & =\bigcup_{m \in \mathbb{N}}\left(-L_{1,1}\right)_{m}=\bigcup_{m \in \mathbb{N}}\left[-\frac{25 \cdot 2^{m-1}+1}{18 \cdot 2^{m-1}},-\frac{25 \cdot 2^{m}+1}{18 \cdot 2^{m}}\right] .
\end{aligned}
$$

By definition, the length of $\left(L_{1,1}\right)_{m}$ and $\left(-L_{1,1}\right)_{m}$ is $\frac{1}{3 \cdot 6 \cdot 2^{m}}$, for each $m \in \mathbb{N}$, then we choose the points $\left(\alpha_{1,1}\right)_{1, m},\left(\alpha_{1,1}\right)_{2, m}$ on $\left(L_{1,1}\right)_{m}$ and, the points $\left(\alpha_{1,1}\right)_{1 m}^{-1},\left(\alpha_{1,1}\right)_{2, m}^{-1}$ on $\left(-L_{1,1}\right)_{m}$, where

$$
\begin{aligned}
& \left(\alpha_{1,1}\right)_{1, m}=\frac{\left(25+6 s_{0}\right) \cdot 2^{m}+1}{3 \cdot 6 \cdot 2^{m}}+\frac{7}{10}\left(\frac{1}{3 \cdot 6 \cdot 2^{m}}\right)=\frac{250 \cdot 2^{m}+17}{180 \cdot 2^{m}}, \\
& \left(\alpha_{1,1}\right)_{2, m}=\frac{\left(25+6 s_{0}\right) \cdot 2^{m}+1}{3.6 \cdot 2^{m}}+\frac{3}{10}\left(\frac{1}{3 \cdot 6 \cdot 2^{m}}\right)=\frac{250 \cdot 2^{m}+13}{180 \cdot 2^{m}}, \\
& \left(\alpha_{1,1}\right)_{1, m}^{-1}=-\frac{250 \cdot 2^{m}+13}{180 \cdot 2^{m}} \\
& \left(\alpha_{1,1}\right)_{2, m}^{-1}=-\frac{250 \cdot 2^{m}+17}{180 \cdot 2^{m}} .
\end{aligned}
$$

For each $m \in \mathbb{N}$, we let $C\left(f_{1,1}\right)_{1, m}, C\left(f_{1,1}\right)_{1, m}^{-1}, C\left(f_{1,1}\right)_{2, m}, C\left(f_{1,1}\right)_{2, m}^{-1}$ be the half-circles having centers $\left(\alpha_{1,1}\right)_{1, m}$,

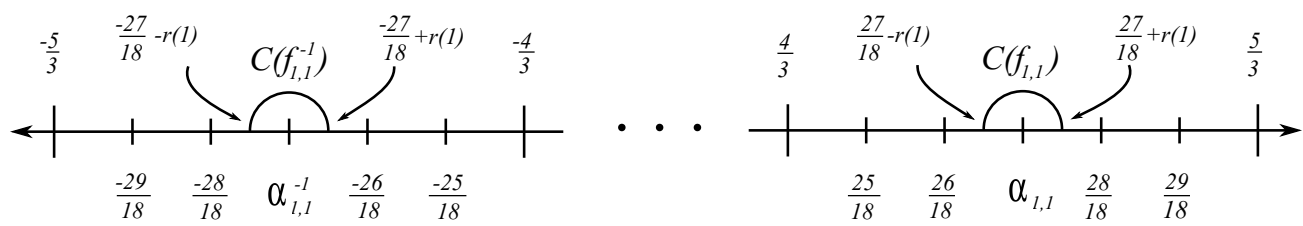

Figure 11: The closed intervals $\hat{I}_{1,1}$ and $-\hat{I}_{1,1}$ are divided into six closed subintervals.

$\left(\alpha_{1,1}\right)_{1, m}^{-1},\left(\alpha_{1,1}\right)_{2, m}$ and $\left(\alpha_{1,1}\right)_{2, m}^{-1}$ respectively, and the same radius $(r(1))_{m}=\frac{1}{10}\left(\frac{1}{3 \cdot 6 \cdot 2^{m}}\right)$ (see Figure 12). Now,

4 These notation were introduced in the step 1 of the proof of the Theorem 1.4. 
we take the Möbius transformations

$$
\left(f_{1,1}\right)_{1, m}(z)=\frac{\left(a_{1,1}\right)_{1, m} z+\left(b_{1,1}\right)_{1, m}}{\left(c_{1,1}\right)_{1, m} z+\left(d_{1,1}\right)_{1, m}}, \quad\left(f_{1,1}\right)_{1, m}^{-1}(z)=\frac{\left(d_{1,1}\right)_{1, m} z-\left(b_{1,1}\right)_{1, m}}{-\left(c_{1,1}\right)_{1, m} z+\left(a_{1,1}\right)_{1, m}},
$$

having as isometric circles $C\left(f_{1,1}\right)_{1, m}$ and $C\left(f_{1,1}\right)_{1, m}^{-1}$, respectively. By Remark 2.9 we have $\left(a_{1,1}\right)_{1, m}=-(250$. $\left.2^{m}+13\right),\left(c_{1,1}\right)_{1, m}=180 \cdot 2^{m},\left(d_{1,1}\right)_{1, m}=-\left(250 \cdot 2^{m}+17\right)$. Now, we substitute these values in the equation $a_{1, m} \cdot d_{1, m}-b_{1, m} \cdot c_{1, m}=1$ and computing we hold $\left(b_{1,1}\right)_{1, m}=\frac{\left(250 \cdot 2^{m}+13\right)\left(250 \cdot 2^{m}+17\right)-1}{180 \cdot 2^{m}}$.

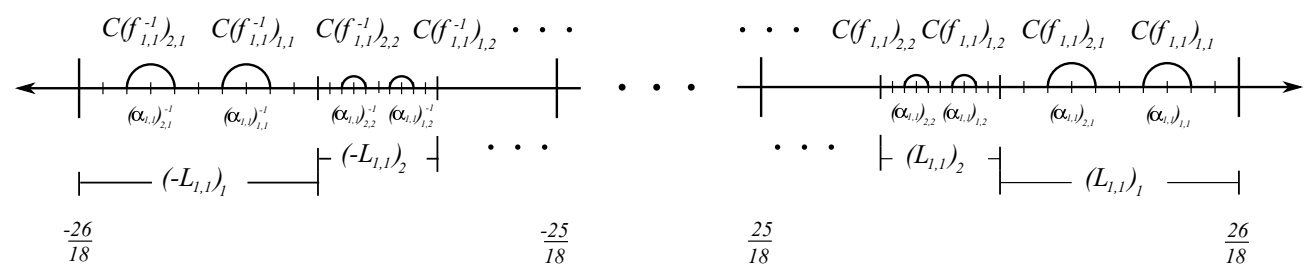

Figure 12: The closed intervals $L_{1,1}$ and $-L_{1,1}$.

On the other hand, for every $m \in \mathbb{N}$, we consider the Möbius transformations

$$
\left(f_{1,1}\right)_{2, m}(z)=\frac{\left(a_{1,1}\right)_{2, m} z+\left(b_{1,1}\right)_{2, m}}{\left(c_{1,1}\right)_{2, m} z+\left(d_{1,1}\right)_{2, m}}, \quad\left(f_{1,1}\right)_{2, m}^{-1}(z)=\frac{\left(d_{1,1}\right)_{2, m} z-\left(b_{1,1}\right)_{2, m}}{-\left(c_{1,1}\right)_{2, m} z+\left(a_{1,1}\right)_{2, m}},
$$

having as isometric circles $C\left(f_{1,1}\right)_{2, m}$ and $C\left(f_{1,1}\right)_{2, m}^{-1}$, respectively. We note that $\left(a_{1,1}\right)_{2, m}=-\left(250 \cdot 2^{m}+17\right)$, $\left(c_{1,1}\right)_{2, m}=180 \cdot 2^{m},\left(d_{1,1}\right)_{2, m}=-\left(250 \cdot 2^{m}+13\right)$ and $\left(b_{1,1}\right)_{2, m}=\frac{\left(250 \cdot 2^{m}+17\right)\left(250 \cdot 2^{m}+13\right)-1}{180 \cdot 2^{m}}$. Then, we define the sets

We remark that by construction each Möbius transformation of the $\left\{\left(f_{1,1}\right)_{1, m},\left(f_{1,1}\right)_{1, m}^{-1}\right.$, $\left.\left(f_{1,1}\right)_{2, m},\left(f_{1,1}\right)_{2, m}^{-1}: m \in \mathbb{N}\right\}$ is hyperbolic and the half-circles of $\left\{C\left(f_{1,1}\right)_{1, m}, C\left(f_{1,1}\right)_{1, m}^{-1}, C\left(f_{1,1}\right)_{2, m}, C\left(f_{1,1}\right)_{2, m}^{-1}\right.$ : $m \in \mathbb{N}\}$ are pairwise disjoint.

Part II. Building sequences of half-circle at the right of $C\left(f_{1,1}\right)$ and at the left of $C\left(f_{1,1}^{-1}\right)$. We consider the fifth sixth closed subintervals of $\hat{I}_{1,1}$ and $-\hat{I}_{1,1}$, which are given by $S_{1,1}=\left[\frac{3+S_{1}}{3}-\frac{2}{3 \cdot 6}, \frac{3+S_{1}}{3}+\frac{1}{3 \cdot 6}\right]=\left[\frac{28}{18}, \frac{29}{18}\right]$ and $-S_{1,1}=\left[-\frac{29}{18},-\frac{28}{18}\right]$, respectively. Now, we write $S_{1,1}$ and $-S_{1,1}$ as the union of closed subintervals following

$$
\begin{aligned}
S_{1,1} & =\bigcup_{m \in \mathbb{N}}\left(S_{1,1}\right)_{m}=\bigcup_{m \in \mathbb{N}}\left[\frac{17+6 S_{1}}{3 \cdot 6}-\frac{1}{3 \cdot 6 \cdot 2^{m-1}}, \frac{17+6 S_{1}}{3 \cdot 6}-\frac{1}{3 \cdot 6 \cdot 2^{m}}\right], \\
& =\bigcup_{m \in \mathbb{N}}\left[\frac{29 \cdot 2^{m-1}-1}{3 \cdot 6 \cdot 2^{m-1}}, \frac{29 \cdot 2^{m}-1}{3 \cdot 6 \cdot 2^{m}}\right], \\
-S_{1,1} & =\bigcup_{m \in \mathbb{N}}\left(-S_{1,1}\right)_{m}=\bigcup_{m \in \mathbb{N}}\left[-\frac{29 \cdot 2^{m-1}-1}{3 \cdot 6 \cdot 2^{m-1}},-\frac{29 \cdot 2^{m}-1}{3 \cdot 6 \cdot 2^{m}}\right] .
\end{aligned}
$$

By definition the length of $\left(S_{1,1}\right)_{m}$ and $\left(-S_{1,1}\right)_{m}$ is $\frac{1}{3 \cdot 6 \cdot 2^{m}}$, for each $m \in \mathbb{N}$, then we choose the points $\left(\alpha_{1,1}\right)_{3, m}$ and $\left(\alpha_{1,1}\right)_{4, m}$ on $\left(S_{1,1}\right)_{m}$, and the points $\left(\alpha_{1,1}\right)_{3, m}^{-1}$ and $\left(\alpha_{1,1}\right)_{4, m}^{-1}$ on $\left(-S_{1,1}\right)_{m}$, where

$$
\begin{aligned}
& \left(\alpha_{1,1}\right)_{3, m}=\frac{\left(17+6 s_{1}\right) \cdot 2^{m-1}-1}{3 \cdot 6 \cdot 2^{m-1}}+\frac{7}{10}\left(\frac{1}{3 \cdot 6 \cdot 2^{m}}\right)=\frac{290 \cdot 2^{m}-13}{180 \cdot 2^{m}}, \\
& \left(\alpha_{1,1}\right)_{4, m}=\frac{\left(170+60 s_{1}\right) \cdot 2^{m-1}-1}{3 \cdot 6 \cdot 2^{m-1}}+\frac{3}{10}\left(\frac{1}{3 \cdot 6 \cdot 2^{m}}\right)=\frac{290 \cdot 2^{m}-17}{180 \cdot 2^{m}}, \\
& \left(\alpha_{1,1}\right)_{3, m}^{-1}=-\frac{290 \cdot 2^{2}-17}{180 \cdot 2^{m}}, \\
& \left(\alpha_{1,1}\right)_{4, m}^{-1}=-\frac{290 \cdot 2^{2}-13}{180 \cdot 2^{m}} .
\end{aligned}
$$

Then we let $C\left(f_{1,1}\right)_{3, m}, C\left(f_{1,1}\right)_{3, m}^{-1}, C\left(f_{1,1}\right)_{4, m}, C\left(f_{1,1}\right)_{4, m}^{-1}$ be the half-circles having centers $\left(\alpha_{1,1}\right)_{3, m},\left(\alpha_{1,1}\right)_{3, m}^{-1}$, $\left(\alpha_{1,1}\right)_{4, m}$ and $\left(\alpha_{1,1}\right)_{4, m}^{-1}$ respectively, and the same radius $(r(1))_{m}=\frac{1}{10}\left(\frac{1}{3 \cdot 6 \cdot 2^{m}}\right)$ (see Figure 13). Now, for every $m \in \mathbb{N}$ we take the Möbius transformations

$$
\left(f_{1,1}\right)_{3, m}(z)=\frac{\left(a_{1,1}\right)_{3, m} z+\left(b_{1,1}\right)_{3, m}}{\left(c_{1,1}\right)_{3, m} z+\left(d_{1,1}\right)_{3, m}}, \quad\left(f_{1,1}\right)_{3, m}^{-1}(z)=\frac{\left(d_{1,1}\right)_{3, m} z-\left(b_{1,1}\right)_{3, m}}{-\left(c_{1,1}\right)_{3, m} z+\left(a_{1,1}\right)_{3, m}},
$$


having as isometric circles $C\left(f_{1,1}\right)_{3, m}$ and $C\left(f_{1,1}\right)_{3, m}^{-1}$. By Remark 2.9 we have $\left(a_{1,1}\right)_{3, m}=-\left(\left(170+60 s_{1}\right) \cdot 2^{m}-\right.$ $17)=-\left(290 \cdot 2^{m}-17\right),\left(c_{1,1}\right)_{3, m}=10 \cdot 3 \cdot 6 \cdot 2^{m}=180 \cdot 2^{m},\left(d_{1,1}\right)_{3, m}=-\left(\left(170+60 s_{1}\right) \cdot 2^{m}-13\right)=-\left(290 \cdot 2^{m}-13\right)$. Now, we substitute these values in equation $\left(a_{1,1}\right)_{3, m} \cdot\left(d_{1,1}\right)_{3, m}-\left(b_{1,1}\right)_{3, m} \cdot\left(c_{1,1}\right)_{3, m}=1$ and computing we hold $\left(b_{1,1}\right)_{3, m}=\frac{\left(290 \cdot 2^{m}-17\right)\left(290 \cdot 2^{m}-13\right)-1}{180 \cdot 2^{m}}$.

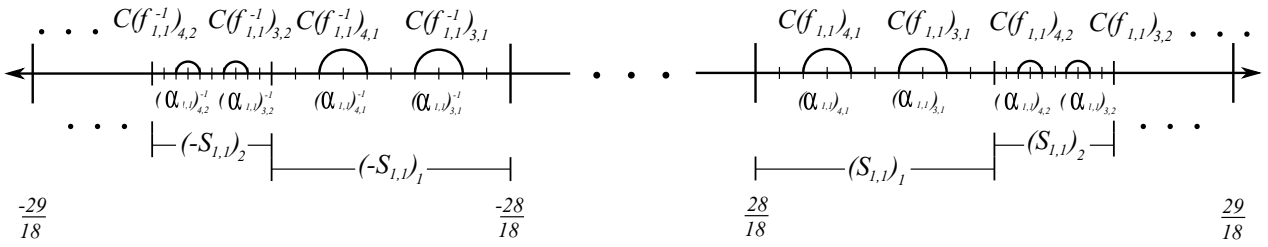

Figure 13: The closed intervals $S_{1,1}$ and $-S_{1,1}$.

In a similar way, for every $m \in \mathbb{N}$ we take the Möbius transformations

$$
\left(f_{1,1}\right)_{4, m}(z)=\frac{\left(a_{1,1}\right)_{4, m} z+\left(b_{1,1}\right)_{4, m}}{\left(c_{1,1}\right)_{4, m} z+\left(d_{1,1}\right)_{4, m}}, \quad\left(f_{1,1}\right)_{4, m}^{-1}(z)=\frac{\left(d_{1,1}\right)_{4, m} z-\left(b_{1,1}\right)_{4, m}}{-\left(c_{1,1}\right)_{4, m} z+\left(a_{1,1}\right)_{4, m}},
$$

having as isometric circles $C\left(f_{1,1}\right)_{4, m}$ and $C\left(f_{1,1}\right)_{4, m}^{-1}$, where $\left(a_{1,1}\right)_{4, m}=-\left(\left(170+60 s_{1}\right) \cdot 2^{m}-13\right)=-(290$. $\left.2^{m}-13\right),\left(c_{1,1}\right)_{4, m}=10 \cdot 3 \cdot 6 \cdot 2^{m}=180 \cdot 2^{m},\left(d_{1,1}\right)_{4, m}=-\left(\left(170+60 s_{1}\right) \cdot 2^{m}-17\right)=-\left(290 \cdot 2^{m}-17\right)$ and $\left(b_{1,1}\right)_{4, m}=\frac{\left(290 \cdot 2^{m}-13\right)\left(290 \cdot 2^{m}-17\right)-1}{180 \cdot 2^{m}}$.

We remark that by construction each Möbius transformation of the set $\left\{\left(f_{1,1}\right)_{3, m},\left(f_{1,1}\right)_{3, m}^{-1}\right.$, $\left.\left(f_{1,1}\right)_{4, m},\left(f_{1,1}\right)_{4, m}^{-1}: m \in \mathbb{N}\right\}$ is hyperbolic and, the half-circles of set $\left\{C\left(f_{1,1}\right)_{3, m}, C\left(f_{1,1}\right)_{3, m}^{-1}\right.$, $\left.C\left(f_{1,1}\right)_{4, m}, C\left(f_{1,1}\right)_{4, m}^{-1}: m \in \mathbb{N}\right\}$ are pairwise disjoint.

Finally, from the equations (11), (13), (14), (16) and (17) we define the sets

$$
\begin{aligned}
& J_{1}=\left\{f_{1,1}, f_{1,1}^{-1},\left(f_{1,1}\right)_{s, m},\left(f_{1,1}\right)_{s, m}^{-1}: s \in\{1, \ldots, 4\}, m \in \mathbb{N}\right\}, \\
& \mathcal{C}_{1}=\left\{C\left(f_{1,1}\right), C\left(f_{1,1}^{-1}\right), C\left(f_{1,1}\right)_{s, m}, C\left(f_{1,1}\right)_{s, m}^{-1}: s \in\{1, \ldots, 4\}, m \in \mathbb{N}\right\} .
\end{aligned}
$$

The elements of $J_{1}$ are hyperbolic and the half-circles of $\mathcal{C}_{1}$ are pairwise disjoint.

For $n$. Building the set $J_{n}$ containing infinitely countable Möbius transformation and the set $\mathcal{C}_{n}$ composed by its respective isometric circles. We let $C\left(f_{n, k}\right), C\left(f_{n, k}^{-1}\right)$ be the half-circles having centers $\alpha_{n, k}$ and $\alpha_{n, k}^{-1}$ respectively (see equation (8)), and the same radius $r(n)=\frac{1}{3^{n \cdot 6 \cdot 2}}$. Now, for each $k \in\left\{0, \ldots, 2^{n-1}-1\right\}$ the values of the coefficients of the Möbius transformations

$$
f_{n, k}(z)=\frac{a_{n, k} z+b_{n, k}}{c_{n, k} z+d_{n, k}}, \quad f_{n, k}^{-1}(z)=\frac{d_{n, k} z-b_{n, k}}{-c_{n, k} z+a_{n, k}},
$$

which has as isometric circles $C\left(f_{n, k}\right)$ and $C\left(f_{n, k}^{-1}\right)$ are $a_{n, k}=-6 \cdot\left(3^{n} \cdot 2+2\left(1+s_{2 k-1}\right)+1\right), c_{n, k}=6 \cdot 3^{n} \cdot 2$, $d_{n, k}=-6 \cdot\left(3^{n} \cdot 2+2\left(1+s_{2 k-1}\right)+1\right)$, and $b_{n, k}=\frac{6^{2} \cdot\left(3^{n} \cdot 2+2\left(1+s_{2 k-1}\right)+1\right)^{2}-1}{6 \cdot 3^{n} \cdot 2}$ (see Figure 14).

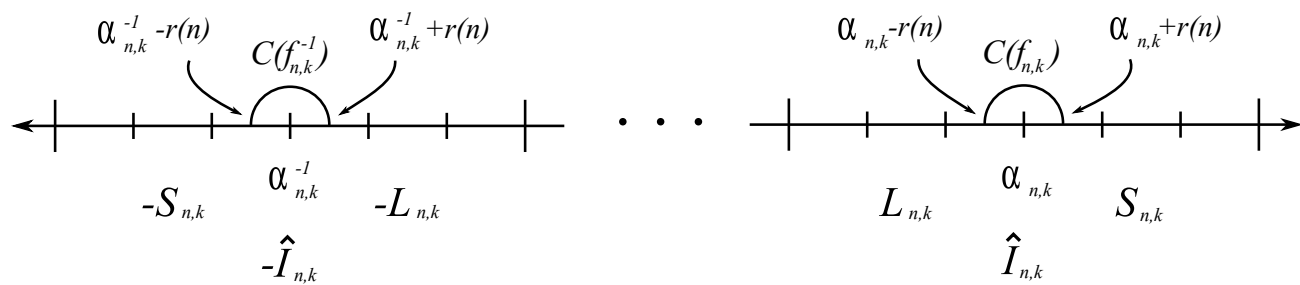

Figure 14: The closed intervals $\hat{I}_{n, k}$ and $-\hat{I}_{n, k}$ are divided into six closed intervals.

By construction the Möbius transformations of the set $\left\{f_{n, k}, f_{n, k}^{-1}: k \in\left\{0, \ldots, 2^{n-1}-1\right\}\right\}$ are hyperbolic and the half-circles of the set $\left\{C\left(f_{n, k}\right), C\left(f_{n, k}^{-1}\right): k \in\left\{0, \ldots, 2^{n-1}-1\right\}\right\}$ are pairwise disjoint. 
Now, for each $k \in\left\{0, \ldots, 2^{n-1}-1\right\}$ we shall build sequences of half-circles at the left and at the right of $C\left(f_{n, k}\right), C\left(f_{n, k}^{-1}\right)$, whose radius converge to zero, each sequence will have associated a suitable sequence of Möbius transformations as follows.

Part I. Building sequences of half-circles at the left of $C\left(f_{n, k}\right)$ and at the right of $C\left(f_{n, k}^{-1}\right)$. For every $k \in\left\{0, \ldots, 2^{n-1}-1\right\}$, we consider the second sixth closed subinterval of the closed intervals $\hat{I}_{n, k}$ and $-\hat{I}_{n, k}$, which are given by

$$
\begin{aligned}
L_{n, k} & =\left[\frac{3^{n}+S_{2 k-2}+1}{3^{n}}+\frac{1}{3^{n} \cdot 6}, \frac{3^{n}+s_{2 k-2}+1}{3^{n}}+\frac{2}{3^{n} \cdot 6}\right], \\
-L_{n, k} & =\left[-\frac{3^{n}+s_{2 k-2}+1}{3^{n}}-\frac{2}{3^{n} \cdot 6},-\frac{3^{n}+s_{2 k-2}+1}{3^{n}}-\frac{1}{3^{n} \cdot 6}\right] .
\end{aligned}
$$

Now, we write $L_{n, k}$ and $-L_{n, k}$ as the union of closed subintervals following

$$
\begin{aligned}
L_{n, k} & =\bigcup_{m \in \mathbb{N}}\left(L_{n, k}\right)_{m}=\bigcup_{m \in \mathbb{N}}\left[\frac{3^{n}+s_{2 k-2}+1}{3^{n}}+\frac{1}{3^{n} \cdot 6 \cdot 2^{m}}, \frac{3^{n}+s_{2 k-2}+1}{3^{n}}+\frac{1}{3^{n} \cdot 6 \cdot 2^{m+1}}\right], \\
& =\left[\frac{6 \cdot 2^{m}\left(3^{n}+s_{2 k-2}+1\right)+1}{3^{n} \cdot 6 \cdot 2^{m}}, \frac{6 \cdot 2^{m+1}\left(3^{n}+S_{2 k-2}+1\right)+1}{3^{n} \cdot 6 \cdot 2^{m+1}}\right] \\
-L_{n, k} & =\bigcup_{m \in \mathbb{N}}\left(-L_{n, k}\right)_{m}=\bigcup_{m \in \mathbb{N}}\left[-\frac{6 \cdot 2^{m+1}\left(3^{n}+s_{2 k-2}+1\right)+1}{3^{n} \cdot 6 \cdot 2^{m+1}},-\frac{6 \cdot 2^{m}\left(3^{n}+s_{2 k-2}+1\right)+1}{3^{n \cdot 6 \cdot 2^{m}}}\right] .
\end{aligned}
$$

By definition the length of $\left(L_{n, k}\right)_{m}$ and $\left(-L_{n, k}\right)_{m}$ is $\frac{1}{3^{n} \cdot 6 \cdot 2^{m}}$, for each $m \in \mathbb{N}$, then we choose the points $\left(\alpha_{n, k}\right)_{1, m},\left(\alpha_{n, k}\right)_{2, m}$ on $\left(L_{n, k}\right)_{m}$, and the points $\alpha_{1, m}^{-1}$ and $\alpha_{2, m}^{-1}$ on $\left(-L_{n, k}\right)_{m}$, where

$$
\begin{aligned}
& \left(\alpha_{n, k}\right)_{1, m}=\frac{3^{n}+s_{2 k-2}+1}{3^{n}}+\frac{1}{3^{n} \cdot 6 \cdot 2^{m}}+\frac{7}{10}\left(\frac{1}{3^{n} \cdot 6 \cdot 2^{m}}\right)=\frac{10 \cdot 6 \cdot 2^{m}\left(3^{n}+s_{2 k-2}+1\right)+17}{3^{n} \cdot 10 \cdot 6 \cdot 2^{m}}, \\
& \left(\alpha_{n, k}\right)_{2, m}=\frac{3^{n}+s_{2 k-2}+1}{3^{n}}+\frac{1}{3^{n} \cdot 6 \cdot 2^{m}}+\frac{3}{10}\left(\frac{1}{3^{n} \cdot 6 \cdot 2^{m}}\right)=\frac{10 \cdot 6 \cdot 2^{m}\left(3^{n}+s_{2 k-2}+1\right)+13}{3^{n} \cdot 10 \cdot 6 \cdot 2^{m}}, \\
& \left(\alpha_{n, k}\right)_{1, m}^{-1}=-\frac{10 \cdot 6 \cdot 2^{m}\left(3^{n}+s_{2 k-2}+1\right)+13}{3^{n} \cdot 10 \cdot 6 \cdot 2^{m}}, \\
& \left(\alpha_{n, k}\right)_{2, m}^{-1}=-\frac{10 \cdot 6 \cdot 2^{m}\left(3^{n}+s_{2 k-2}+1\right)+17}{3^{n} \cdot 10 \cdot 6 \cdot 2^{m}} .
\end{aligned}
$$

Then we let $C\left(f_{n, k}\right)_{1, m}, C\left(f_{n, k}\right)_{1, m}^{-1}, C\left(f_{n, k}\right)_{2, m}, C\left(f_{n, k}\right)_{2, m}^{-1}$ be the half-circles having centers $\left(\alpha_{n, k}\right)_{1, m},\left(\alpha_{n, k}\right)_{1, m}^{-1}$, $\left(\alpha_{n, k}\right)_{2, m}$ and $\left(\alpha_{n, k}\right)_{2, m}^{-1}$, respectively, and the same radius $(r(n))_{m}=\frac{1}{10}\left(\frac{1}{3^{n} \cdot 6 \cdot 2^{m}}\right)$ (see Figure 15). Now, for every $m \in \mathbb{N}$ the values of the coefficients of the Möbius transformations

$$
\left(f_{n, k}\right)_{1, m}(z)=\frac{\left(a_{n, k}\right)_{1, m} z+\left(b_{n, k}\right)_{1, m}}{\left(c_{n, k}\right)_{1, m} z+\left(d_{n, k}\right)_{1, m}}, \quad\left(f_{n, k}\right)_{1, m}^{-1}(z)=\frac{(d n, k)_{1, m} z-\left(b_{n, k}\right)_{1, m}}{-\left(c_{n, k}\right)_{1, m} z+\left(a_{n, k}\right)_{1, m}},
$$

having as isometric circles $C\left(f_{n, k}\right)_{1, m}$ and $C\left(f_{n, k}\right)_{1, m}^{-1}$, respectively, are $\left(a_{n, k}\right)_{1, m}=-\left(10 \cdot 6 \cdot 2^{m}\left(3^{n}+s_{2 k-2}+\right.\right.$ $1)+13),\left(c_{n, k}\right)_{1, m}=10 \cdot 3^{n} \cdot 6 \cdot 2^{m},\left(d_{n, k}\right)_{1, m}=-\left(10 \cdot 6 \cdot 2^{m}\left(3^{n}+s_{2 k-2}+1\right)+17\right)$, and $\left(b_{n, k}\right)_{1, m}=$ $\frac{\left(10 \cdot 6 \cdot 2^{m}\left(3^{n}+s_{2 k-2}+1\right)+13\right)\left(10 \cdot 6 \cdot 2^{m}\left(3^{n}+s_{2 k-2}+1\right)+17\right)-1}{10 \cdot 3^{n} \cdot 6 \cdot 2^{m}}$.

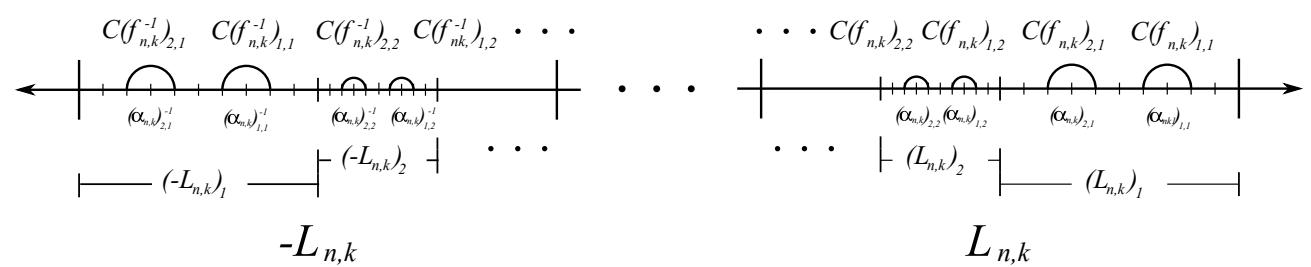

Figure 15: The closed intervals $-L_{n, k}$ and $L_{n, k}$.

By construction each Möbius transformation of the set $\left\{\left(f_{n, k}\right)_{1, m},\left(f_{n, k}\right)_{1, m}^{-1},\left(f_{n, k}\right)_{2, m},\left(f_{n, k}\right)_{2, m}^{-1}: m \in\right.$ $\left.\mathbb{N}, k \in\left\{0, \ldots, 2^{n-1}-1\right\}\right\}$ is hyperbolic and the half-circle of set $\left\{C\left(f_{n, k}\right)_{1, m}, C\left(f_{n, k}\right)_{1, m}^{-1}, C\left(f_{n, k}\right)_{2, m}, C\left(f_{n, k}\right)_{2, m}^{-1}\right.$ : $\left.m \in \mathbb{N}, k \in\left\{0, \ldots, 2^{n-1}-1\right\}\right\}$ are pairwise disjoint.

Part II. Building sequences of half-circle on the right $C\left(f_{n, k}\right)$ and at the left $C\left(f_{n, k}^{-1}\right)$. For each $k \in$ $\left\{0, \ldots, 2^{n-1}-1\right\}$ we consider the fifth sixth closed subintervals $\hat{I}_{n, k}$ and $-\hat{I}_{n, k}$, which are given by

$$
\begin{aligned}
S_{n, k} & =\left[\frac{3^{n}+S_{2 k-1}}{3^{n}}-\frac{2}{3^{n} \cdot 6}, \frac{3^{n}+S_{2 k-1}}{3^{n}}-\frac{1}{3^{n} \cdot 6}\right]=\left[\frac{6\left(3^{n}+S_{2 k-1}\right)-2}{3^{n} \cdot 6}, \frac{6\left(3^{n}+S_{2 k-1}\right)-1}{3^{n} \cdot 6}\right], \\
-S_{n, k} & =\left[-\frac{6\left(3^{n}+S_{2 k-1}\right)-2}{3^{n} \cdot 6},-\frac{6\left(3^{n}+S_{2 k-1}\right)-1}{3^{n} \cdot 6}\right] .
\end{aligned}
$$


Then we write $S_{n, k}$ and $-S_{n, k}$ as the union of closed subintervals following

$$
\begin{aligned}
S_{n, k} & =\bigcup_{m \in \mathbb{N}}\left(S_{n, k}\right)_{m}=\bigcup_{m \in \mathbb{N}}=\left[\frac{6\left(3^{n}+S_{2 k-1}\right)-1}{3^{n \cdot 6}}-\frac{1}{3^{n} \cdot 6 \cdot 2^{m-1}}, \frac{6\left(3^{n}+s_{2 k-1}\right)-1}{3^{n} \cdot 6}-\frac{1}{3^{n} \cdot 6 \cdot 2^{m}}\right], \\
& =\bigcup_{m \in \mathbb{N}}\left[\frac{2^{m-1}\left(6\left(3^{n}+3_{2 k-1}\right)-1\right)-1}{3^{n} \cdot 6 \cdot 2^{m-1}}, \frac{2^{m}\left(6\left(3^{n}+S_{2 k-1}\right)-1\right)-1}{3^{n} \cdot 6 \cdot 2^{m}}\right], \\
-S_{n, k} & =\bigcup_{m \in \mathbb{N}}\left(-S_{n, k}\right)_{m}=\bigcup_{m \in \mathbb{N}}\left[-\frac{2^{m}\left(6\left(3^{n}+s_{2 k-1}\right)-1\right)-1}{3^{n} \cdot 6 \cdot 2^{m}},-\frac{2^{m}\left(6\left(3^{n}+s_{2 k-1}\right)-1\right)-1}{3^{n} \cdot 6 \cdot 2^{m}}\right] .
\end{aligned}
$$

By definition the length of $\left(S_{n, k}\right)_{m}$ and $\left(-S_{n, k}\right)_{m}$ is $\frac{1}{3^{n} \cdot 6 \cdot 2^{m}}$, for each $m \in \mathbb{N}$, then we choose the points $\left(\alpha_{n, k}\right)_{3, m}$ and $\left(\alpha_{n, k}\right)_{4, m}$ on $\left(S_{n, k}\right)$, the points $\left(\alpha_{n, k}\right)_{3, m}^{-1, k}$ and $\left(\alpha_{n, k}\right)_{4, m}^{-1}$ on $\left(-S_{n, k}\right)_{m}$, where

$$
\begin{aligned}
& \left(\alpha_{n, k}\right)_{3, m}=\frac{2^{m-1}\left(6\left(3^{n}+s_{2 k-1}\right)-1\right)-1}{3^{n} \cdot 6 \cdot 2^{m-1}}+\frac{7}{10}\left(\frac{1}{3^{n} \cdot 6 \cdot 2^{m}}\right)=\frac{2^{m}\left(6\left(3^{n}+s_{2 k-1}\right)-1\right)-13}{3^{n} \cdot 6 \cdot 10 \cdot 2^{m}}, \\
& \left(\alpha_{n, k}\right)_{4, m}=\frac{2^{m-1}\left(6\left(3^{n}+s_{2 k-1}\right)-1\right)-1}{3^{n} \cdot 6 \cdot \cdot^{m-1}}+\frac{3}{10}\left(\frac{1}{3^{n} \cdot 6 \cdot 2^{m}}\right)=\frac{2^{m}\left(6\left(3^{n}+s_{2 k-1}\right)-1\right)-17}{3^{n} \cdot 6 \cdot 10 \cdot 2^{m}} . \\
& \left(\alpha_{n, k}\right)_{3, m}^{-1}=-\frac{2^{m}\left(6\left(3^{n}+s_{2 k-1}\right)-1\right)-17}{3^{n} \cdot 6 \cdot 10 \cdot 2^{m}}, \\
& \left(\alpha_{n, k}\right)_{4, m}^{-1}=-\frac{2^{m}\left(6\left(3^{n}+s_{2 k-1}\right)-1\right)-13}{3^{n} \cdot 6 \cdot 10 \cdot 2^{m}} .
\end{aligned}
$$

Then we let $C\left(f_{n, k}\right)_{3, m}, C\left(f_{n, k}\right)_{3, m}^{-1}, C\left(f_{n, k}\right)_{4, m}, C\left(f_{n, k}\right)_{4, m}^{-1}$ be the half-circles having centers $\left(\alpha_{n, k}\right)_{3, m},\left(\alpha_{n, k}\right)_{3, m}^{-1}$, $\left(\alpha_{n, k}\right)_{4, m}$ and $\left(\alpha_{n, k}\right)_{4, m}^{-1}$, respectively, and the same radius $(r(n))_{m}=\frac{1}{10}\left(\frac{1}{3^{n} \cdot 6 \cdot 2^{m}}\right)$ (see Figure 16).

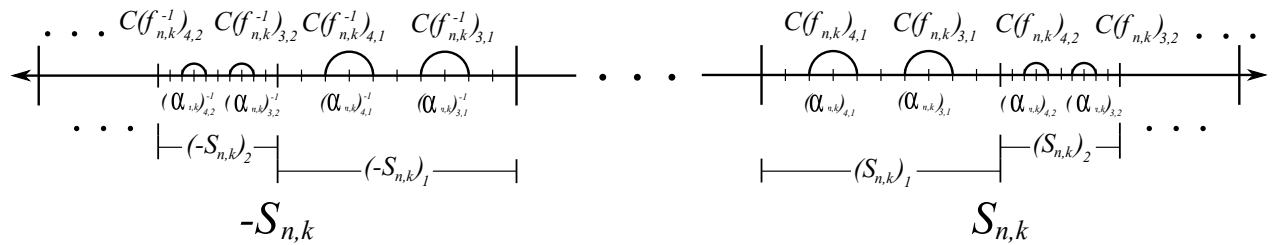

Figure 16: The closed intervals $S_{n, k}$ and $-S_{n, k}$.

Then, for every $m \in \mathbb{N}$, the values of the coefficients of the Möbius transformations

$$
\left(f_{n, k}\right)_{3, m}(z)=\frac{\left(a_{n, k}\right)_{3, m} z+\left(b_{n, k}\right)_{3, m}}{\left(c_{n, k}\right)_{3, m} z+\left(d_{n, k}\right)_{3, m}}, \quad\left(f_{n, k}\right)_{3, m}^{-1}(z)=\frac{\left(d_{n, k}\right)_{3, m} z-\left(b_{n, k}\right)_{3, m}}{-\left(c_{n, k}\right)_{3, m} z+\left(a_{n, k}\right)_{3, m}},
$$

having as isometric circles $C\left(f_{n, k}\right)_{3, m}$ and $C\left(f_{n, k}\right)_{3, m}^{-1}$, respectively, are $\left(a_{n, k}\right)_{3, m}=-\left(2^{m}\left(6\left(3^{n}+s_{2 k-1}\right)-\right.\right.$ 1) - 13), $\left(c_{n, k}\right)_{3, m}=10 \cdot 3^{n} \cdot 6 \cdot 2^{m},\left(d_{n, k}\right)_{3, m}=-\left(2^{m}\left(6\left(3^{n}+s_{2 k-1}\right)-1\right)-17\right)$, and $\left(b_{n, k}\right)_{3, m}=$ $\frac{\left(2^{m}\left(6\left(3^{n}+s_{2 k-1}\right)-1\right)-13\right)\left(2^{m}\left(6\left(3^{n}+s_{2 k-1}\right)-1\right)-17\right)-1}{10 \cdot 3^{n} \cdot 6 \cdot 2^{m}}$.

On the other hand, for every $m \in \mathbb{N}$, the values of the coefficients of the Möbius transformations

$$
\left(f_{n, k}\right)_{4, m}(z)=\frac{\left(a_{n, k}\right)_{4, m} z+\left(b_{n, k}\right)_{4, m}}{\left(c_{n, k}\right)_{4, m} z+\left(d_{n, k}\right)_{4, m}}, \quad\left(f_{n, k}\right)_{4, m}^{-1}(z)=\frac{\left(d_{n, k}\right)_{4, m} z-\left(b_{n, k}\right)_{4, m}}{-\left(c_{n, k}\right)_{4, m} z+\left(a_{n, k}\right)_{4, m}},
$$

having as isometric circles $C\left(f_{n, k}\right)_{4, m}$ and $C\left(f_{n, k}\right)_{4, m}^{-1}$, respectively, are $\left(a_{n, k}\right)_{4, m}=-\left(2^{m}\left(6\left(3^{n}+s_{2 k-1}\right)-\right.\right.$ 1) - 13), $\left(c_{n, k}\right)_{4, m}=10 \cdot 3^{n} \cdot 6 \cdot 2^{m},\left(d_{n, k}\right)_{4, m}=-\left(2^{m}\left(6\left(3^{n}+s_{2 k-1}\right)-1\right)-17\right)$, and $\left(b_{n, k}\right)_{4, m}=$ $\frac{\left(2^{m}\left(6\left(3^{n}+s_{2 k-1}\right)-1\right)-13\right)\left(2^{m}\left(6\left(3^{n}+s_{2 k-1}\right)-1\right)-17\right)-1}{10 \cdot 3^{n} \cdot 6 \cdot 2^{m}}$.

By construction each Möbius transformation of the set $\left\{\left(f_{n, k}\right)_{3, m},\left(f_{n, k}\right)_{3, m}^{-1},\left(f_{n, k}\right)_{4, m},\left(f_{n, k}\right)_{4, m}^{-1}: m \in\right.$ $\left.\mathbb{N}, k \in\left\{0, \ldots, 2^{n-1}-1\right\}\right\}$ is hyperbolic and the half-circle of set $\left\{C\left(f_{n, k}\right)_{3, m}, C\left(f_{n, k}\right)_{3, m}^{-1}, C\left(f_{n, k}\right)_{4, m}, C\left(f_{n, k}\right)_{4, m}^{-1}\right.$ : $\left.m \in \mathbb{N}, k \in\left\{0, \ldots, 2^{n-1}-1\right\}\right\}$ are pairwise disjoint.

Finally, from the equations (19), (21), (24) and (25) we define the sets of the Möbius maps and their respective isometric circles by

$$
\begin{aligned}
& J_{n}=\left\{f_{n, k},\left(f_{n, k}\right)_{s, m}(z): k \in\left\{0, \ldots, 2^{n-1}-1\right\}, s \in\{1, \ldots, 4\}, m \in \mathbb{N}\right\}, \\
& \mathcal{C}_{n}=\left\{C\left(f_{n, k}\right), C\left(f_{n, k}^{-1}\right), C\left(f_{n, k}\right)_{s, m}, C\left(f_{n, k}\right)_{s, m}^{-1}: k \in\left\{0, \ldots, 2^{n-1}-1\right\}, s \in\{1, \ldots, 4\}, m \in \mathbb{N}\right\} .
\end{aligned}
$$


We remark that by construction each Möbius transformation of $J_{n}$ is hyperbolic and the half-circles of $\mathcal{C}_{n}$ are pairwise disjoint.

By the previous recursive construction of Möbius transformations and half-circles we define the sets

$$
J:=\bigcup_{n \in \mathbb{N}} J_{n}, \quad \mathcal{C}:=\bigcup_{n \in \mathbb{N}} \mathcal{C}_{n},
$$

and we denote as $\Gamma$ the subgroup of $P S L(2, \mathbb{R})$ generated by the union $J$. We note that by construction each Möbius transformation of $J$ is hyperbolic and the half-circle of $\mathcal{C}$ are pairwise disjoint.

Step 2. The group $\Gamma$ is a Fuchsian group. In order to $\Gamma$ will be a Geometric Schottky group, we shall define a Schottky description for it. Hence, by Proposition 2.17 conclude that $\Gamma$ is Fuchsian.

The elements belonged to the set $J$ can be indexed by a symmetric subset of $\mathbb{Z}$. Merely, we let $P:=\left\{p_{n}\right\}_{n \in \mathbb{N}}$ be the subset of $\mathbb{N}$ composed by all the primes numbers, then is easy to check that the map $\psi: J \rightarrow \mathbb{Z}$ such that

$$
f_{n, k} \mapsto p_{4+n}^{k}, \quad f_{n, k}^{-1} \mapsto-p_{4+n}^{k}, \quad\left(f_{n, k}\right)_{s, m} \mapsto p_{s}^{m} \cdot p_{4+n}^{k}, \quad\left(f_{n, k}\right)_{s, m}^{-1} \mapsto-p_{s}^{m} \cdot p_{4+n}^{k},
$$

for every $n, m \in \mathbb{N}, k \in\left\{0, \ldots, 2^{n-1}-1\right\}, s \in\{1, \ldots, 4\}$, it is well-defined and injective. We note that the image of $J$ under $\psi$ is a symmetric subset of $\mathbb{Z}$, which we denote as $I$. Given that for each element $k$ belonged to $I$ there is a unique transformation $f \in J$, such that $\psi(f)=k$, we label the map $f$ as $f_{k}$ and its respective isometric circle $C(f)$ as $C\left(f_{k}\right)$. Hence, we re-write the sets $J$ and $\mathcal{C}$ as

$$
J=\left\{f_{k}\right\}_{k \in I}, \quad \mathcal{C}=\left\{C\left(f_{k}\right)\right\}_{k \in I} .
$$

On the other hand, we define the set $\left\{A_{k}\right\}_{k \in I}$ where $A_{k}$ is the straight segment in the real line $\mathbb{R}$ whose ends points coincide with the endpoints at infinite of the half-circle $C\left(f_{k}\right)$ (see equation (27)). We claim that the pair $\left(\left\{A_{k}\right\},\left\{f_{k}\right\}\right)_{k \in I}$ is a Schottky description. Regarding the recursive construction of the family $J=\left\{f_{k}\right\}_{k \in I}$ described above, it is immediate that the pair $\left(\left\{A_{k}\right\},\left\{f_{k}\right\}\right)_{f_{k} \in J}$ satisfies the conditions 1 and 2 of Definition 2.14. Thus, we must only prove that the fact 3 is done. The proof is the same as in the Cantor tree case.

Step 3. Holding the surface called the Blooming Cantor tree. The Geometric Schottky group $\Gamma$ acts freely and properly discontinuously on $\mathbb{H}$, because the intersection of any two different elements of $\mathcal{C}$ is empty. Then the quotient space $S=\mathbb{H} / \Gamma$ is a well-defined and through the projection map $\pi: \mathbb{H} \rightarrow S$ is a hyperbolic surface. Following the same ideas that in the Cantor tree (surface) case we conclude that $S$ has ends space homeomorphic to the Cantor set and, by construction each end has infinite genus.

Corollary 3.4. The fundamental group of the Blooming Cantor tree is isomorphic to $\Gamma$.

Corollary 3.5. The fundamental group of the Cantor tree is isomorphic to any subgroup of the fundamental group of the blooming Cantor tree.

Acknowledgements: The authors sincerely thank Jesús Muciño Raymundo, Rubén Antonio Hidalgo Ortega, and Fernando Hernández Hernández for their constructive conversations and valuable help. The second author was partially supported by UNIVERSIDAD NACIONAL DE COLOMBIA, SEDE MANIZALES. Camilo Ramírez Maluendas have dedicated this work to his beautiful family: Marbella and Emilio, in appreciation of their love and support.

\section{References}

[1] William Abikoff, The uniformization theorem, Amer. Math. Monthly 88 (1981), no. 8, 574-592.

[2] Alan F. Beardon, A Premier on Riemann Surfaces, London Mathematical Society Lecture Note Series, vol. 78, Cambridge University Press, Cambridge, 1984.

[3] __ The geometry of discrete groups, Graduate Texts in Mathematics, vol. 91, Springer-Verlag, New York, 1983.

[4] J. Cantwell and L. Conlon, Leaves with isolated ends in foliated 3-manifolds, Topology 16 (1977), no. 4, $311-322$. 
[5] James Dugundji, Topology, Allyn and Bacon, Inc., Boston, Mass.-London-Sydney, 1978. Reprinting of the 1966 original; Allyn and Bacon Series in Advanced Mathematics.

[6] Henri de Saint-Gervais, Uniformization of Riemann Surfaces Revisiting a hundred-year-old theorem, European Mathematical Society, 2010.

[7] H. M. Farkas and I Kra, Riemann Surfaces, 2nd ed., Graduate Texts in Mathematics, vol. 71, Springer-Verlag, New York, 1992.

[8] L. R. Ford, The fundamental region for a Fuchsian group, Bull. Amer. Math. Soc. 31 (1925), no. 9-10, 531-539.

[9] Hans Freudenthal, Über die Enden topologischer Räume und Gruppen, Math. Z. 33 (1931), no. 1, 692-713 (German).

[10] Étienne Ghys, Topologie des feuilles génériques, Ann. of Math. (2) 141 (1995), no. 2, 387-422 (French).

[11] Svetlana Katok, Fuchsian groups, Chicago Lectures in Mathematics, University of Chicago Press, Chicago, IL, 1992.

[12] __ Fuchsian groups, geodesic flows on surfaces of constant negative curvature and symbolic coding of geodesics, Homogeneous flows, moduli spaces and arithmetic, Clay Math. Proc., vol. 10, Amer. Math. Soc., Providence, RI, 2010, pp. $243-320$.

[13] Béla. Kerékjártó, Vorlesungen über Topologie I, Mathematics: Theory \& Applications, Springer, Berlín, 1923.

[14] John M. Lee, Introduction to topological manifolds, Graduate Texts in Mathematics, vol. 202, Springer-Verlag, New York, 2000.

[15] Bernard Maskit, Kleinian groups, Grundlehren der Mathematischen Wissenschaften [Fundamental Principles of Mathematical Sciences], vol. 287, Springer-Verlag, Berlin, 1988.

[16] Dúwang Prada, A golden Cantor Set, Undergrade Dissertation, Industrial University of Santander, Bucaramanga, Colombia, 2006 (Spanish).

[17] C. Ramírez Maluendas and F. Valdez, Veech group of infinite genus surfaces, Algebr. Geom. Topol. 17 (2017), no. 1, 529-560.

[18] Frank Raymond, The end point compactification of manifolds, Pacific J. Math. 10 (1960), 947-963.

[19] Ian Richards, On the classification of noncompact surfaces, Trans. Amer. Math. Soc. 106 (1963), 259-269.

[20] Richard Evan Schwartz, Mostly surfaces, Student Mathematical Library, vol. 60, American Mathematical Society, Providence, RI, 2011.

[21] Ernst Specker, Die erste Cohomologiegruppe von Überlagerungen und Homotopie-Eigenschaften dreidimensionaler Mannigfaltigkeiten, Comment. Math. Helv. 23 (1949), 303-333 (German).

[22] Stephen Willard, General topology, Addison-Wesley Publishing Co., Reading, Mass.-London-Don Mills, Ont., 1970.

[23] Anna M. Zielicz, Geometry and dynamics of infinitely generated Kleinian groups-Geometrics Schottky groups, PhD Dissertation, Universität Bremen, 2015. 